\title{
SCIENTIFIC REPORTS

\section{Quaternary ice sheets and sea level regression drove divergence in a marine gastropod along Eastern and Western coasts of South} America

\author{
P. J. Fernández Iriarte $\mathbb{1}^{1}$, C. A. González-Wevar $\mathbb{1}^{2,3,4^{*}}$, N. I. Segovia ${ }^{4,5}$, S. Rosenfeld ${ }^{6}$, \\ M. Hüne ${ }^{4}$, L. Fainburg ${ }^{1}$, J. D. Nuñez ${ }^{1}$, P. A. Haye ${ }^{5}$ \& E. Poulin $\mathbb{1}^{4}$
}

The southern coastline of South America is a remarkable area to evaluate how Quaternary glacial processes impacted the demography of the near-shore marine biota. Here we present new phylogeographic analyses in the pulmonate Siphonaria lessonii across its distribution, from northern Chile in the Pacific to Uruguay in the Atlantic. Contrary to our expectations, populations from the southwestern Atlantic, an area that was less impacted by ice during glacial maxima, showed low genetic diversity and evidence of recent expansion, similar to the patterns recorded in this study across heavily ice-impacted areas in the Pacific Magellan margin. We propose that Atlantic and Pacific shallow marine hard-substrate benthic species were both affected during the Quaternary in South America, but by different processes. At higher latitudes of the southeast Pacific, ice-scouring drastically affected S. lessonii populations compared to non-glaciated areas along the Chile-Peru province where the species was resilient. In the southwest Atlantic, S. lessonii populations would have been dramatically impacted by the reduction of near-shore rocky habitat availability as a consequence of glacio-eustatic movements. The increase of gravelly and rocky shore substrates in the southwest Atlantic supports a hypothesis of glacial refugia from where the species recolonized lower latitudes across the Atlantic and Pacific margins. Our results suggest that current patterns of genetic diversity and structure in near-shore marine benthic species do not solely depend on the impact of Quaternary glacial ice expansions but also on the availability of suitable habitats and life-history traits, including developmental mode, bathymetry and the likelihood of dispersal by rafting.

Quaternary ice sheet expansion and contraction during the Last Glacial Maximum (LGM), between 23,000 and 18,000 years before present, triggered major climate and environmental changes that strongly affected the distribution of the biota worldwide ${ }^{1,2}$. Under the Expansion-Contraction (E-C) model of Pleistocene biogeography, cold-temperate species contracted their distribution ranges towards lower latitude glacial refugia located in less ice-impacted or non-glaciated areas ${ }^{3}$. During interglacial periods they recolonized higher latitudes through range expansions following deglaciation processes ${ }^{4-6}$. This simple E-C model provides a straightforward paradigm to test population demographic hypotheses through the Quaternary, and phylogeographic studies have helped to understand better the response of species to major climate changes during this period by recognizing distribution range shifts, potential refugial areas and recolonization routes $^{7-9}$.

${ }^{1}$ IIMyC, Instituto de Investigaciones Marinas y Costeras, CONICET - FCEyN, Universidad Nacional de Mar del Plata, Funes 3250 (7600), Mar del Plata, Provincia de Buenos Aires, Argentina. ${ }^{2}$ Laboratorio de Genómica y Ecología Molecular Antártica y sub-Antártica, Instituto de Ciencias Marinas y Limnológicas (ICML), Universidad Austral de Chile, Casilla \# 567, Valdivia, Chile. ${ }^{3}$ Centro FONDAP de Investigación en Dinámica de Ecosistemas Marinos de Altas Latitudes (IDEAL), Universidad Austral de Chile, Casilla \# 567, Valdivia, Chile. ${ }^{4}$ Instituto de Ecología y Biodiversidad (IEB), Departamento de Ciencias Ecológicas, Facultad de Ciencias, Universidad de Chile, Las Palmeras \#, 3425, Ñuñoa, Santiago, Chile. ${ }^{5}$ Laboratorio de Diversidad Molecular, Departamento de Biología Marina, Facultad de Ciencias del Mar, Universidad Católica del Norte, Coquimbo, Chile. ${ }^{6}$ Laboratorio de Ecosistemas Marinos Antárticos y Subantárticos, Universidad de Magallanes, Bulnes, 01890, Punta Arenas, Chile. *email: claudio.gonzalez@uach.cl 
In southern South America, glacial periods produced major shifts in sea level, climate and landscapes, particularly in marine near-shore environments ${ }^{10}$. During the LGM, the Pacific margin of the Magellan province, from Chiloé Island $\left(42^{\circ} \mathrm{S}\right)$ to Cape Horn $\left(56^{\circ} \mathrm{S}\right)$, was almost completely covered by the Patagonian Ice Sheet ${ }^{10-12}$. Consequently, the availability of near-shore sheltered and hard-substrate habitats in the Pacific Magellan margin became very limited or even absent, and accordingly impeded the persistence of numerous near-shore marine species. Heavily ice-impacted areas were recolonized as the ice receded by dispersal of individuals from refugial populations. Repetitive rounds of local extinctions followed by recolonization may have enhanced the diversification of invertebrates across the region by the availability/diversity of vacant habitats and/or by survival in multiple refugia that may have led to allopatric divergence ${ }^{13,14}$.

In contrast to the glacial history recorded along the Pacific Magellan margin, ice sheet expansion and contraction would have affected the marine ecosystems across the southwestern Atlantic to a much lesser extent ${ }^{15}$. Accordingly, near-shore marine species in the Atlantic Magellan margin could have persisted in situ during the last glacial period, in contrast to their conspecific populations located in the Pacific.

During the last two decades phylogeographic and population-based studies have been carried out for different groups of Magellan freshwater ${ }^{16,17}$ and near-shore marine ${ }^{18-24}$ organisms. Phylogeographic studies in freshwater and terrestrial Magellan ecosystems have inferred the presence of Quaternary glacial refugia on the east side of the Andes $^{25}$, as well as on the Pacific Magellan margin within ${ }^{17,26}$ and outside the glacier limits ${ }^{19,27}$. Phylogeographic studies in Magellan marine organisms have established that most of the taxa analyzed exhibited low levels of genetic diversity, absence of genetic structure and strong signals of recent demographic growth, supporting the hypothesis of postglacial expansions ${ }^{19-23,27,28}$. Nevertheless, although several near-shore marine species are broadly distributed across the whole Magellan province, very few phylogeographic studies have been conducted across the whole region ${ }^{21,22,29}$. In fact, most of the studies have been restricted either to the Pacific ${ }^{18,19,29,30}$ or to the Atlantic ${ }^{23,31}$ margin of the Magellan province. Therefore, a phylogeographic study conducted on a broadly distributed species would represent an important contribution to propose an integrated scenario of how coastal marine biota have responded to historical climate changes along temperate and cold areas of southern South America.

Members of the pulmonate genus Siphonaria are distributed worldwide, except in the Arctic. In the last revision of the genus, Dayrat et al. ${ }^{32}$ recognized three distinct species in southern South America: Siphonaria lessonii Blainville, 1827, S. lateralis Gould, 1846, and the recently described S. fuegiensis ${ }^{33}$. Siphonaria lessonii is commonly found on intertidal rocky ecosystems and is widely distributed from southern Peru to Cape Horn in the Pacific and north to Uruguay in the Atlantic, as well as in the Malvinas/Falkland Islands ${ }^{34}$. Accordingly, the distribution of this pulmonate limpet extends across different ecoregions ${ }^{35}$ in temperate areas of the Chile-Peru and the Argentina provinces, as well as cold areas across the Magellan Province ${ }^{36}$. The species S. lessonii is a hermaphroditic broadcast-spawner with free-swimming veliger larvae that disperse for approximately 8 to 11 days in the water column ${ }^{37}$. Considering the geographic range and the reproductive biology of the species, S. lessonii is a suitable model to infer the impact of Quaternary glacial cycles over its distribution range, demographic inferences, recolonization pathways and its local persistence across a latitudinal gradient in areas that were differentially affected by the Quaternary glacial processes.

A first phylogeographic genetic approach based on a fragment of the mitochondrial DNA (mtDNA) COI gene recognized the presence of two genetic clades in the species, one on the Pacific margin of South America and one on the Atlantic side, which would have diverged between 100,000 and 1,000,000 years ago ${ }^{24}$. However, the low number of samples from the Pacific margin did not allow them to locate the actual limit between the recorded genetic clades. More recently, new phylogeographic analyses in the species were combined with environmental niche modelling analyses along the southeast Pacific coast ${ }^{28}$. The results of this study are congruent with a historical scenario involving Quaternary demographic and distribution contractions of S. lessonii surviving in glacial refugia in the southern portion of the southeastern Pacific, followed by recolonization of the deglaciated areas. Nevertheless, the absence of information concerning the genetic diversity of the species along its Atlantic range may result in an incomplete or misinterpreted Quaternary demographic scenario. Therefore, a broader phylogeographic study of $S$. lessonii is required to better understand the historical biogeography of a species widely distributed across the southern Atlantic and Pacific coasts of South America.

This study aims to evaluate the impacts of past glaciations on marine taxa using as a model species the pulmonate Siphonaria lessonii across different South American provinces. For this purpose we performed phylogeographic and population-based analyses using fragments of the mitochondrial cytochrome oxidase $c$ subunit 1 (COI) and the nuclear internal transcribed spacers (ITS1 and ITS2). We hypothesize that Quaternary glacial processes have differentially affected $S$. lessonii, since habitat eradication would have strongly impacted populations along Pacific coasts. In contrast, southwestern Atlantic populations may have persisted through glacial cycles, as previously demonstrated in the Chile-Peru Province. Accordingly, populations from the Magallanes Province, and particularly those from the Pacific margin are expected to show lower levels of genetic diversity than temperate populations from the Chile-Peru and Argentina provinces, and should harbor a genetic signal of a recent demographic expansion process. Conversely, lower latitude populations located in non-glaciated areas of the southwestern Atlantic and the Chile-Peru province are expected to exhibit higher levels of genetic diversity and structure as a consequence of more stable demographic histories. Hence, we expect to find different genetic signatures between higher latitude Magellan populations and lower latitude temperate populations located across the Atlantic and Pacific South American margins.

\section{Results}

Sample collection and genetic polymorphism in S. lessonii. Siphonaria lessonii individuals were collected from 25 localities across the species' distribution in three main South American biogeographic areas as defined by Trovant et al. ${ }^{29}$ (Fig. 1). 


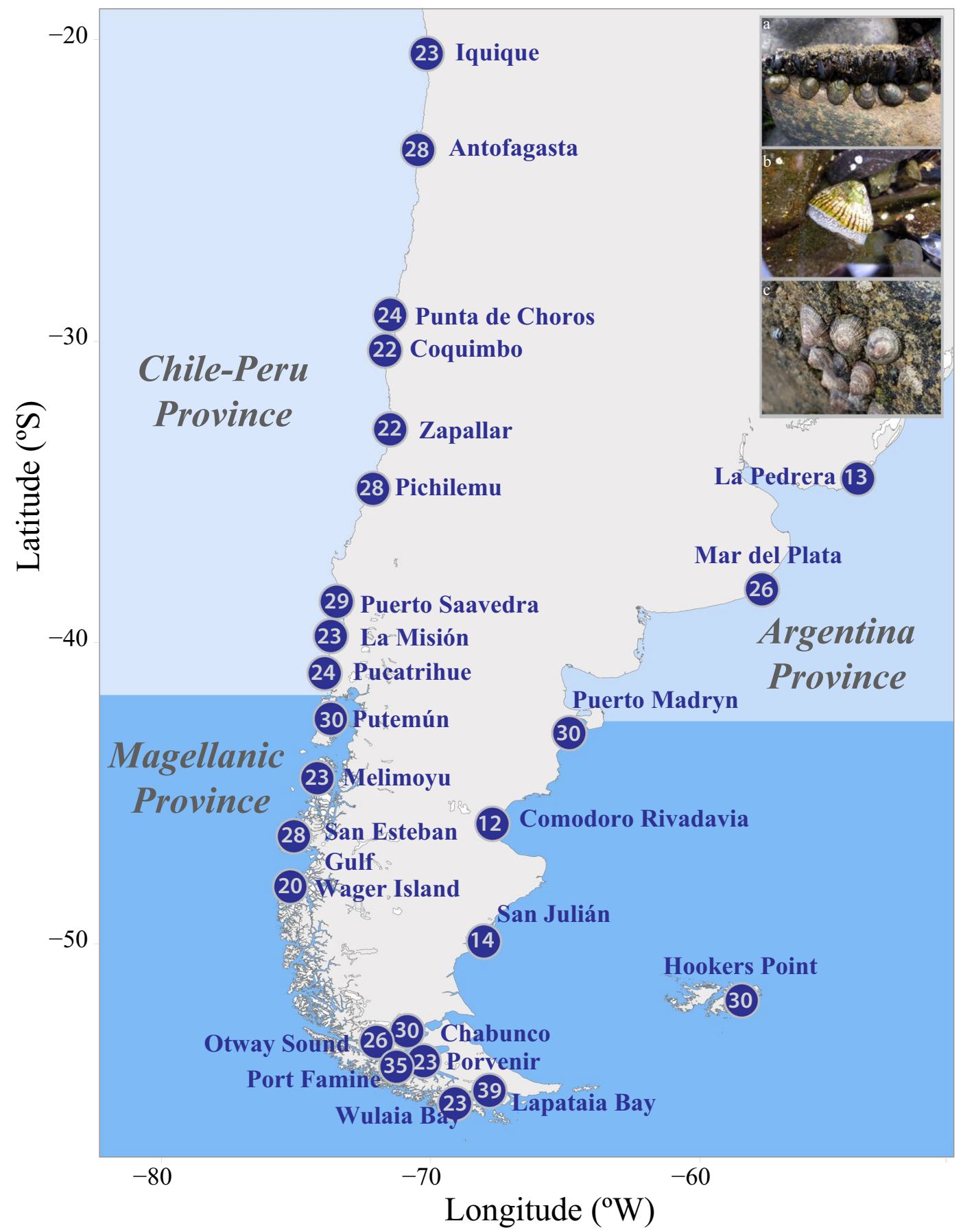

Figure 1. Sampling localities of Siphonaria lessonii across its distribution in different provinces of the southeastern Pacific and the southwestern Atlantic. Shapefiles of the coastlines available in the database of GEOdas (NOAA) were filtered using GEOdas Coastline Extractor v. 1.1.3.1. (https://www.ngdc.noaa.gov/ mgg/geodas/geodas.html). Photographs of intertidal S. lessonii courtesy of co-authors Pilar Haye (a \& b) and Sebastian Rosenfeld (c).

The complete COI data set of S. lessonii included 625 individuals and consisted of 565 nucleotide positions coding for 188 amino acids. Out of the analyzed COI sequences, 563 are new for the species and the rest $(n=62)$ were included in the study of Nuñez et al. ${ }^{25}$ No insertion, deletion, or stop codons were detected in the whole COI data set. The complete ITS data set included 45 new sequences, consisted of 1259 nucleotides and a total of six insertion/deletions were recorded. Mitochondrial and nuclear sequences were not saturated at any position and within the COI data set we recorded four amino acid substitutions (positions 6, 31, 100, and 117). High levels of mtDNA genetic variability characterized S. lessonii with 115 polymorphic positions (20.35\%), of which 66 (57.39\%) were parsimoniously informative. Mitochondrial DNA sequences were A-T rich (63.5\%) compared to the mean G-C content. Conversely, nuclear ITS sequences were G-C rich (59.3\%) and more conserved 


\begin{tabular}{|l|l|l|l|l|}
\hline Source of variation & d.f. & Sum of squares & $\begin{array}{l}\text { Variance } \\
\text { components }\end{array}$ & $\begin{array}{l}\text { Percentage of } \\
\text { variation }\end{array}$ \\
\hline Among groups & 1 & 810.655 & $2.81539 \mathrm{Va}$ & 64.48 \\
\hline Among populations within groups & 23 & 68.000 & $0.05881 \mathrm{Vb}$ & 1.35 \\
\hline Within populations & 600 & 895.460 & $1.49243 \mathrm{Vc}$ & 34.18 \\
\hline Total & 624 & 1774.115 & 4.36663 & \\
\hline
\end{tabular}

Table 1. Spatial Analysis of Molecular Variance (SAMOVA) across Siphonaria lessonii's distribution depicting the percentage of variation explained among groups (C1 and C2) among populations within groups, and within populations. $\mathrm{F}_{\mathrm{SC}}=$ differentiation within populations among groups and $\mathrm{F}_{\mathrm{CT}}=$ differentiation among groups $(* * \mathrm{p}<0.01, * * * \mathrm{p}<0.001) . \mathrm{R} 1=$ Chile-Peru Province; $\mathrm{R} 2=$ Magellan Province + Argentina Province. Fixation Index $\mathrm{F}_{\mathrm{SC}}: 0.03791 * * * \mathrm{~F}_{\mathrm{CT}}: 0.64475 * * *$.

than mtDNA sequences, with only 24 polymorphic positions (1.90\%) of which $13(54.1 \%)$ were parsimoniously informative.

Main patterns of genetic structure in S. lessonii across its distribution. SAMOVA and GENELAND analyses recovered similar patterns of phylogeographic structure in S. lessonii, with the presence of two main genetic groups. SAMOVA recovered two significantly differentiated groups $\left(\mathrm{F}_{\mathrm{CT}}=0.64\right)$ explaining $64.48 \%$ of the total variance. In contrast, differences between localities within groups were significant $\left(\mathrm{F}_{\mathrm{SC}}=0.03\right)$ but only explained $1.35 \%$ of the total variation (Table 1). The first genetic group (C1) included populations along the Chile-Peru Province (CP) north of $42^{\circ} \mathrm{S}$, between Iquique $\left(20^{\circ} \mathrm{S}\right)$ and Pucatrihue $\left(40^{\circ} \mathrm{S}\right)$. The second genetic group (C2) included sites located south of $42^{\circ} \mathrm{S}$ along the Magellan province together with localities from the Argentina province, as well as the Malvinas/Falkland Islands (MFI). The Bayesian clustering algorithm detected the same phylogeographic structure with the recognition of two main groups $(\mathrm{K}=2)(\mathrm{Fig} .2 \mathrm{~A}, \mathrm{~B})$. Values for group membership were very high for all localities (c.a. $\mathrm{P}=0.95)$. The mean probability value $(\mathrm{P}=0.5)$, which corresponds to the boundary between these genetic groups, crossed the Pacific coast at approximately $42^{\circ} \mathrm{S}$ (Fig. 2). Again, these two genetic groups included 1) CP localities and 2) MP + MFI and AP localities (Fig. 2). The nearest neighbor statistic algorithm in the complete $S$. lessonii COI data set $\left(\mathrm{S}_{\mathrm{nn}}=0.11\right)$ detected a low but significant phylogeographic signal $(\mathrm{P}<0.001)$. However, when this analysis was performed considering the SAMOVA and GENELAND groupings $(\mathrm{C} 1$ vs. $\mathrm{C} 2), \mathrm{S}_{\mathrm{nn}}$ became very high $\left(\mathrm{S}_{\mathrm{nn}}=0.87\right)$ and remained highly significant $(\mathrm{P}<0.0001)$, demonstrating the high phylogeographic signal detected in the species across its distribution.

Contrasting patterns of genetic diversity structure in S. lessonii across its distribution. Levels of mtDNA genetic diversity were significantly higher in R1 populations across the Chile-Peru province (CP) compared to those recorded south of $42^{\circ} \mathrm{S}$, across MP and AP within R2 (Table 2). Extremely low levels of genetic diversity recorded at La Pedrera, Uruguay, Comodoro Rivadavia, and San Julián, Argentina, must be taken with precaution considering the reduced number of individuals analyzed in these sites $(12,14$, and 13 respectively, Table 2). Accordingly, these localities were excluded from subsequent comparative analyses. Haplotype diversity $(H)$ varied between 1.000 (Iquique, CP) and 0.418 (Hookers Point, MFI). The number of polymorphic sites $(S)$ varied between 27 (Iquique, CP) and 7 (Wulaia Bay, MP). Similarly, the number of haplotypes $(k$ ) varied between 30 (Iquique, CP) and 6 (Wager Island and Porvenir, MP) (Table 2). The average number of nucleotide differences $(\Pi)$, and the mean nucleotide diversity $(\pi)$ varied between $6.53 / 0.0115$ (La Misión, CP) and 0.6/0.001 (Wulaia, MP) (Table 2).

Considering the high levels of genetic diversity recorded in R1 populations and the high number of single-frequency haplotypes recorded in this genetic group, the analyses of structure were performed using mean pairwise differences $\left(\mathrm{N}_{\mathrm{ST}}\right)$. Mean general values of differentiation were high, especially considering the mean $\mathrm{N}_{\mathrm{ST}}=0.314$ (Table 3). Nevertheless, most of the differences were found comparing R1 vs. R2 localities. Sites across the Chile-Peru province (R1) showed low levels of genetic differentiation from Iquique (20 $\left.{ }^{\circ} \mathrm{S}\right)$ to Pucatrihue $\left(40^{\circ} \mathrm{S}\right)$ (Table 3). Similarly, comparisons between R2 populations failed to recognize any significant structure in $S$. lessonii south of $42^{\circ} \mathrm{S}$ from Chiloé in the Pacific to Uruguay in the Atlantic (Table 3).

Genealogical reconstructions in S. lessonii. The maximum parsimony network of mtDNA COI sequences in S. lessonii recorded a total of 192 haplotypes (Fig. 3) and clearly discriminated two main genetic clusters that are separated by six substitution steps (Fig. 3). The first cluster (C1) was mainly formed by individuals collected in R1 (91.1\%) localities along CP, but some individuals (8.9\%) from R2 (MP) also fell within C1. Similarly, the second cluster (C2) consisted of individuals from MP and AP but some individuals (9.2\%) from R1 (CP) also fell within $\mathrm{C} 2$. Interestingly, the percentage of R1 individuals recorded in $\mathrm{C} 2$, as well as the percentage of R2 individuals collected in $\mathrm{C} 1$ decreased as we move away from the cluster's boundary, located around $42^{\circ} \mathrm{S}$ (Figs. 3, S2 and S3).

As previously determined using standard diversity indices, $\mathrm{C} 1$ along the $\mathrm{CP}$ showed a more expanded genealogy than C2 across MP and AP. Several medium-frequency haplotypes (H01-H04) in C1 were broadly distributed across $\mathrm{CP}$ from Iquique $\left(20^{\circ} \mathrm{S}\right)$ to Pucatrihue $\left(40^{\circ} \mathrm{S}\right)$. Associated with these medium-frequency haplotypes we recorded many low- to single-frequency ones $(\mathrm{n}=114)$. Conversely, the $\mathrm{C} 2$ parsimony network showed a typical star-like topology with a very short genealogy (Fig. 3). A central dominant haplotype (H05) was the most frequent in the whole data set (44\%) and mainly distributed across MP and AP (Fig. 3). According to Posada and Crandall ${ }^{38}$, this dominant haplotype should represent the most ancestral of the Magellan diversity, whereas 


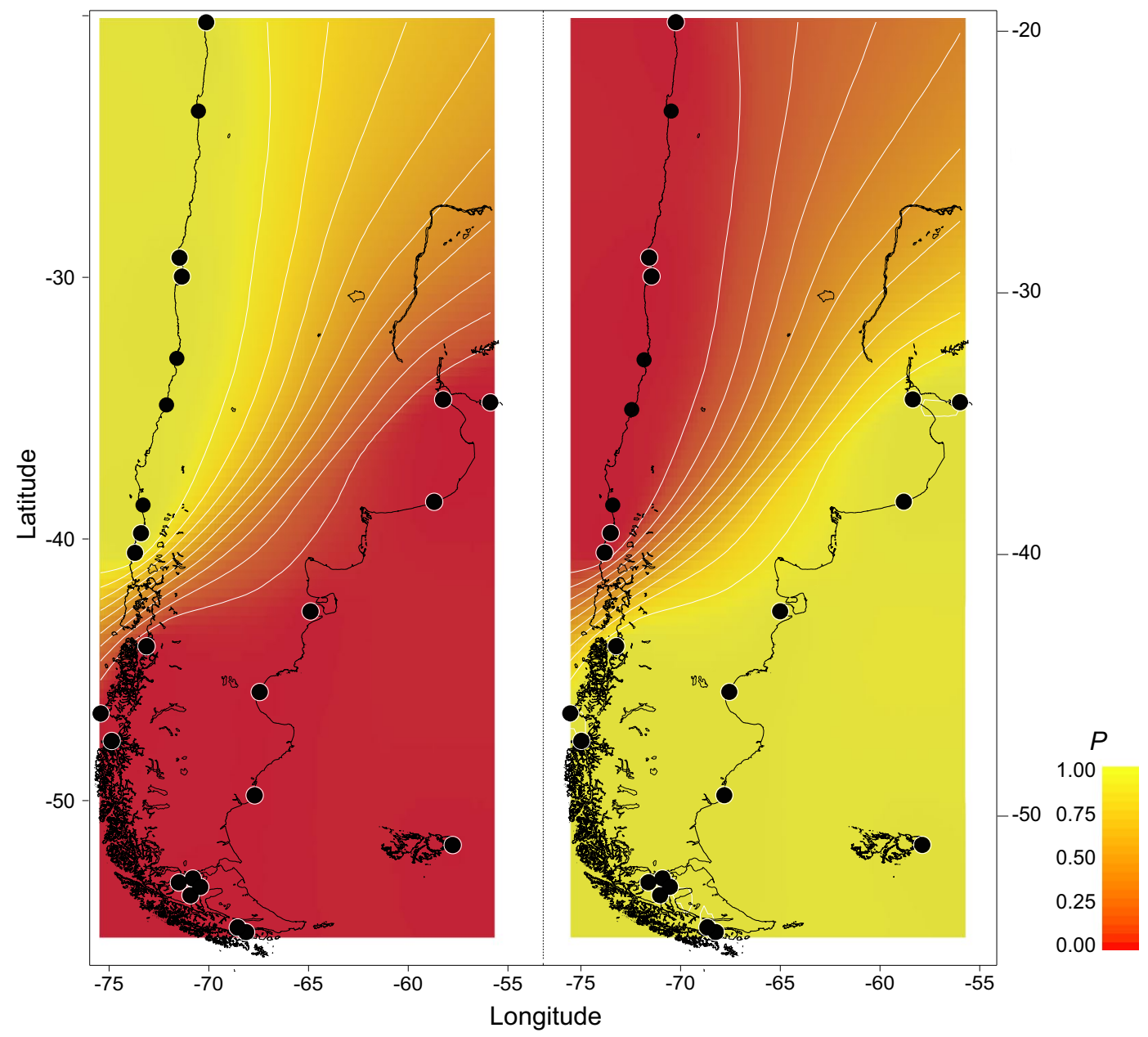

Figure 2. Spatial genetic structure of Siphonaria lessonii across its distribution on the southeastern Pacific and the southwestern Atlantic based on GENELAND analyses. The black circles indicate the relative positions of the sampling localities. Dark and light shades are proportional to posterior probabilities of membership in clusters. Yellow and red areas show the highest and lowest probabilities of membership in clusters, respectively. Posterior probabilities were plotted against the southern South America coastline shapefile plotted in the database of GEOdas (NOAA) and filtered using GEOdas Coastline Extractor v 1.1.3.1 (https://www.ngdc.noaa.gov/mgg/ geodas/geodas.html).

the most derived C2 haplotype is related to it with a maximum branch length of 5 mutational steps (Fig. 3). A medium-frequency haplotype (H06) recorded in several C2 localities was also detected in one individual from La Misión (CP). Many low- to single-frequency haplotypes surrounded the dominant Magellan haplotype $(\mathrm{n}=72)$ of C2 (Fig. 3). The dominant haplotype recorded in C2 (H05) was also found in several individuals collected at $\mathrm{R} 1$ localities, and it was more frequent in those sites located close to the cluster boundary at $42^{\circ} \mathrm{S}$ (e.g. La Misión and Pucatrihue) (Fig. S2). Similarly, several individuals collected at R2 localities carried medium-frequency haplotypes from the $\mathrm{C} 1$ diversity (H01-H03), particularly in sites located in the MP populations located near the cluster's boundary at $42^{\circ} \mathrm{S}$ (e.g. Putemún, Melimoyu, and San Esteban Gulf).

The nuclear ITS maximum parsimony network included a total of 19 different haplotypes (Fig. S3), with a similar pattern of genetic diversity and structure to those estimated for COI. The nuclear genealogy also discriminated two major genetic clusters, separated by four mutational steps (Fig. S3). Again, C1 included individuals from Iquique to Pucatrihue (PC) that exhibited a more expanded genealogy compared to those from MP and AP populations that showed a typical star-like one. The dominant $\mathrm{C} 1$ haplotype (H01) was widely distributed across the CP. Surrounding H01 we recorded a total of nine single-frequency haplotypes; four of them were separated by more than one mutational step (Fig. S3). A second dominant haplotype (H13) was widely distributed in C2 populations from the MP and AP, including the Malvinas/Falkland Islands. A total of six single-frequency haplotypes are closely associated with this dominant Magellan haplotype. As recorded in COI sequences, one $\mathrm{C} 1$ individual from la Misión in R1 (CP) shared the dominant sequence found in R2 (H13). Similarly, another individual from Melimoyu in R2 (MP) carried the dominant haplotype of C1 (H01) (Fig. S3).

Gene flow and connectivity. Maximum likelihood gene flow analysis using MIGRATE with different migration models detected evidence of low levels of gene flow with signals of being asymmetric from the CP (C1) towards the $\mathrm{MP}+\mathrm{AP}(\mathrm{C} 2)$. Among the tested models, the one from $\mathrm{C} 1$ to $\mathrm{C} 2$ received the highest probability 


\begin{tabular}{|c|c|c|c|c|c|c|c|c|c|c|}
\hline Locality & Lat. & Long. & $N$ & $k$ & $H$ & $S$ & $\Pi$ & $\pi$ & D & $F_{s}$ \\
\hline Iquique & -20.25 & -70.13 & 23 & 23 & 1.000 & 27 & 4.22 & 0.007 & $-1.82^{*}$ & $-26.76^{* * * *}$ \\
\hline Antofagasta & -23.48 & -70.40 & 28 & 21 & 0.976 & 26 & 3.52 & 0.006 & -1.70 & $-16.76^{* * *}$ \\
\hline Punta de Choros & -29.25 & -71.46 & 24 & 17 & 0.938 & 34 & 4.38 & 0.007 & $-1.97^{*}$ & $-8.75 * * *$ \\
\hline Coquimbo & -29.96 & -71.33 & 22 & 14 & 0.948 & 24 & 3.81 & 0.006 & -1.58 & $-5.92 * *$ \\
\hline Zapallar & -32.55 & -72.45 & 22 & 15 & 0.952 & 22 & 3.23 & 0.005 & -1.73 & $-8.73 * * *$ \\
\hline Pichilemu & -34.41 & -72.03 & 28 & 22 & 0.976 & 31 & 5.39 & 0.009 & -1.18 & $-13.69 * * *$ \\
\hline Puerto Saavedra & -38.78 & -73.33 & 29 & 23 & 0.975 & 37 & 5.70 & 0.010 & -1.57 & $-14.39 * * *$ \\
\hline La Misión & -39.78 & -73.40 & 23 & 18 & 0.972 & 29 & 6.53 & 0.011 & -0.64 & $-7.76^{* * *}$ \\
\hline Pucatrihue & -40.53 & -73.70 & 24 & 17 & 0.960 & 23 & 5.56 & 0.009 & -0.35 & $6.82 * *$ \\
\hline Cluster 01 & & & 223 & 111 & 0.977 & 84 & 4.99 & 0.008 & $-2.00^{*}$ & $-158.818^{* * * *}$ \\
\hline Putemún & -42.41 & -73.75 & 30 & 13 & 0.648 & 20 & 3.10 & 0.005 & -1.34 & $-4-04^{*}$ \\
\hline Melimoyu & -44.10 & -75.43 & 23 & 13 & 0.818 & 22 & 5.06 & 0.008 & -0.59 & $-2.69^{*}$ \\
\hline San Esteban Gulf & -46.66 & -74.86 & 28 & 19 & 0.881 & 28 & 5.52 & 0.009 & -0.84 & $-8.04 * * *$ \\
\hline Wager Island & -47.71 & -74.87 & 20 & 6 & 0.516 & 15 & 3.18 & 0.005 & -0.91 & 1.08 \\
\hline Port Famine & -53.61 & -70.92 & 35 & 11 & 0.496 & 20 & 1.62 & 0.002 & $-2-25^{* *}$ & $-4.89 * *$ \\
\hline Otway Sound & -53.13 & -71.50 & 26 & 10 & 0.748 & 11 & 1.20 & 0.002 & $-1.93 *$ & $-6.33^{* *}$ \\
\hline Chabunco & -52.98 & -70.80 & 30 & 10 & 0.517 & 10 & 0.72 & 0.001 & $-2.25 * *$ & $-8.98 * * *$ \\
\hline Porvenir & -53.30 & -70.43 & 23 & 6 & 0.395 & 19 & 2.04 & 0.003 & $-2.21 * *$ & 0.02 \\
\hline Wulaia Bay & -55.03 & -68.13 & 23 & 7 & 0.462 & 7 & 0.60 & 0.001 & $-2.14 * *$ & $-5.25 * *$ \\
\hline Lapataia Bay & -54.85 & -68.55 & 39 & 13 & 0.560 & 14 & 1.00 & 0.001 & $-2.24 * *$ & $-10.65 * * *$ \\
\hline San Julián & -49.80 & -67.70 & 14 & 5 & 0.505 & 11 & 1.81 & 0.003 & $-1.91^{*}$ & 0.020 \\
\hline Comodoro Rivadavia & -45.85 & -67.45 & 12 & 2 & 0.167 & 1 & 0.16 & 0.000 & -1.14 & -0.47 \\
\hline Puerto Madryn & -42.76 & -64.90 & 30 & 9 & 0.632 & 19 & 2.807 & 0.004 & -1.43 & -0.91 \\
\hline Mar del Plata & -38.03 & -57.51 & 26 & 9 & 0.578 & 8 & 0.757 & 0.001 & $-2.18 * *$ & $-7.35^{* * *}$ \\
\hline La Pedrera & -34.58 & 54.11 & 13 & 1 & 0.000 & 0 & 0 & 0 & $\mathrm{n} / \mathrm{a}$ & $\mathrm{n} / \mathrm{a}$ \\
\hline Hookers Point & -51.70 & -57.76 & 30 & 8 & 0.418 & 19 & 1.26 & 0.002 & $-2.55 * * *$ & $-2.97 *$ \\
\hline Cluster 02 & & & 402 & 93 & 0.568 & 78 & 2.13 & 0.003 & $-2.43 * * *$ & $-153.32 * * *$ \\
\hline Total & & & 625 & 151 & 0.749 & 97 & 5.04 & 0.0091 & $-1.94^{*}$ & $-213.80 * * *$ \\
\hline
\end{tabular}

Table 2. Geographical coordinates, diversity indices and neutrality tests (Tajima's D and Fu's $\mathrm{F}_{\mathrm{S}}$ ) in Siphonaria lessonii populations across its distribution in the southeast Pacific and the southwest Atlantic. Where: $\mathrm{n}=$ number of analyzed individuals; $\mathrm{k}=$ number of haplotypes; $\mathrm{S}=$ polymorphic sites; $\mathrm{H}=$ haplotype diversity; $\Pi=$ average number of pairwise differences; $\pi=$ nucleotide diversity. $* \mathrm{p}<0.05,{ }^{*} \mathrm{p}<0.01, * * * \mathrm{p}<0.001$.

(Table 4). Similarly, the coalescent approach of isolation-with-migration implemented in IMA2 detected overall low levels of gene flow between C1 and C2 (Table 5). Nevertheless, the model predicted a most likely pattern of asymmetric migration with high and significant migration from C1 to C2 (LRT, 10.092) (Fig. 4A, Table 5), and low and non-significant migration (LRT, 2.583) from C2 to C1 (Fig. 4A, Table 5). IMA2 analyses also estimated that the separation between C1 and C2 occurred between 100,000 and 200,000 years ago, using a mutation rate between 1 and $2 \%$ (Fig. 4B, Table 5).

Demographic inference in S. lessonii. According to the Bayes factor analysis, the uncorrelated relaxed clock with exponential distribution is the model that best fitted our data with the HKY $+\mathrm{I}+\mathrm{G}$ estimated by jModelTest. Bayesian skyline plot analyses resulted in different demographic trajectories for the genetic clusters recorded in S. lessonii across its distribution in southern South America. Populations in the Chile-Peru province showed a more stable and older demographic history than those of the Magellan and Argentinian provinces, which showed evidence of a very recent expansion (Fig. 5A). Bayesian skyline plot analyses allowed us a rough estimation for the time of the most recent common ancestors (tmrca) and the timing of population expansions between the recorded genetic groups in S. lessonii (Fig. 5A). Time estimates using a population-based mutational rate suggest that the tmrca for $\mathrm{C} 1$ may have occurred around $43 \mathrm{ka}$, while the trmca of $\mathrm{C} 2$ would have occurred about $8 \mathrm{ka}$. Moreover, the onset of population expansion in $\mathrm{C} 1$ was dated around $35 \mathrm{ka}$ while in $\mathrm{C} 2$ it took place approximately $5 \mathrm{ka}$. As expected for star-like genealogies, global Tajima's D and Fu's F neutrality tests were both negative and significant for each of the recognized clusters and for the whole COI data set (Table 2). Considering the main patterns of genetic diversity and structure recorded in S. lessonii, the distribution of pairwise differences varies considerably between the main genetic clusters. The mismatch distribution of $\mathrm{C} 1$ was unimodal (Fig. 5B) while the distribution of pairwise differences in $\mathrm{C} 2$ showed a typical L-shaped distribution (Fig. 5C).

\section{Discussion}

How species and ecosystems responded to Quaternary glacial cycles can tell us about the evolution and biogeography of the biota during the last million years and provide useful information for understanding their responses to future climate change $e^{1-4,7-9,30,40-43}$. In this study we recorded differences in the genetic signals of the pulmonate S. lessonii between temperate and high latitude South American areas, probably associated with contrasting 


\begin{tabular}{|c|c|c|c|c|c|c|c|c|c|c|c|c|c|c|c|c|c|c|c|c|c|c|c|c|c|}
\hline & 1 & 2 & 3 & 4 & 5 & 6 & 7 & 8 & 9 & 10 & 11 & 12 & 13 & 14 & 15 & 16 & 17 & 18 & 19 & 20 & 21 & 22 & 23 & 24 & 25 \\
\hline 1 & $* * *$ & & & & & & & & & & & & & & & & & & & & & & & & \\
\hline 2 & 0.00 & $* * *$ & & & & & & & & & & & & & & & & & & & & & & & \\
\hline 3 & 0.00 & 0.00 & $* * *$ & & & & & & & & & & & & & & & & & & & & & & \\
\hline 4 & 0.00 & 0.00 & 0.00 & **** & & & & & & & & & & & & & & & & & & & & & \\
\hline 5 & 0.00 & 0.00 & 0.00 & 0.00 & $* * *$ & & & & & & & & & & & & & & & & & & & & \\
\hline 6 & 0.02 & 0.01 & 0.00 & 0.00 & 0.00 & $* * *$ & & & & & & & & & & & & & & & & & & & \\
\hline 7 & 0.01 & 0.02 & 0.00 & 0.00 & 0.01 & 0.00 & $* * *$ & & & & & & & & & & & & & & & & & & \\
\hline 8 & 0.10 & 0.10 & 0.06 & 0.00 & 0.05 & 0.01 & 0.01 & $* * *$ & & & & & & & & & & & & & & & & & \\
\hline 9 & 0.07 & 0.08 & 0.05 & 0.06 & 0.03 & 0.00 & 0.00 & 0.00 & **** & & & & & & & & & & & & & & & & \\
\hline 10 & 0.62 & 0.64 & 0.60 & 0.60 & 0.62 & 0.49 & 0.51 & 0.38 & 0.42 & $* * *$ & & & & & & & & & & & & & & & \\
\hline 11 & 0.45 & 0.48 & 0.42 & 0.42 & 0.44 & 0.31 & 0.33 & 0.20 & 0.23 & 0.02 & *** & & & & & & & & & & & & & & \\
\hline 12 & 0.45 & 0.48 & 0.43 & 0.42 & 0.44 & 0.33 & 0.34 & 0.22 & 0.24 & 0.02 & 0.00 & $* * *$ & & & & & & & & & & & & & \\
\hline 13 & 0.60 & 0.63 & 0.58 & 0.59 & 0.61 & 0.47 & 0.48 & 0.35 & 0.39 & 0.00 & 0.01 & 0.01 & $* * *$ & & & & & & & & & & & & \\
\hline 14 & 0.73 & 0.75 & 0.71 & 0.72 & 0.74 & 0.61 & 0.62 & 0.52 & 0.56 & 0.00 & 0.13 & 0.10 & 0.01 & *** & & & & & & & & & & & \\
\hline 15 & 0.75 & 0.77 & 0.73 & 0.75 & 0.77 & 0.64 & 0.64 & 0.54 & 0.59 & 0.05 & 0.19 & 0.15 & 0.06 & 0.00 & *** & & & & & & & & & & \\
\hline 16 & 0.78 & 0.79 & 0.76 & 0.78 & 0.80 & 0.66 & 0.66 & 0.57 & 0.62 & 0.05 & 0.20 & 0.16 & 0.07 & 0.00 & 0.00 & *** & & & & & & & & & \\
\hline 17 & 0.69 & 0.71 & 0.67 & 0.69 & 0.71 & 0.57 & 0.57 & 0.46 & 0.51 & 0.00 & 0.10 & 0.08 & 0.00 & 0.00 & 0.00 & 0.00 & *** & & & & & & & & \\
\hline 18 & 0.77 & 0.79 & 0.75 & 0.77 & 0.79 & 0.65 & 0.65 & 0.56 & 0.60 & 0.06 & 0.20 & 0.15 & 0.08 & 0.00 & 0.00 & 0.00 & 0.01 & $* * *$ & & & & & & & \\
\hline 19 & 0.78 & 0.79 & 0.77 & 0.78 & 0.80 & 0.68 & 0.68 & 0.59 & 0.63 & 0.06 & 0.22 & 0.18 & 0.08 & 0.00 & 0.00 & 0.00 & 0.00 & 0.00 & *** & & & & & & \\
\hline 20 & 0.67 & 0.69 & 0.64 & 0.66 & 0.69 & 0.53 & 0.54 & 0.42 & 0.46 & 0.00 & 0.05 & 0.03 & 0.00 & 0.00 & 0.02 & 0.00 & 0.00 & 0.03 & 0.02 & $* * *$ & & & & & \\
\hline 21 & 0.74 & 0.76 & 0.71 & 0.74 & 0.77 & 0.60 & 0.61 & 0.50 & 0.55 & 0.03 & 0.15 & 0.11 & 0.04 & 0.00 & 0.00 & 0.00 & 0.00 & 0.00 & 0.00 & 0.01 & *** & & & & \\
\hline 22 & 0.66 & 0.68 & 0.63 & 0.64 & 0.66 & 0.54 & 0.54 & 0.43 & 0.46 & 0.00 & 0.07 & 0.05 & 0.00 & 0.00 & 0.03 & 0.03 & 0.00 & 0.04 & 0.04 & 0.00 & 0.01 & $* * *$ & & & \\
\hline 23 & 0.77 & 0.78 & 0.75 & 0.77 & 0.79 & 0.65 & 0.65 & 0.56 & 0.60 & 0.05 & 0.19 & 0.15 & 0.07 & 0.00 & 0.01 & 0.00 & 0.00 & 0.00 & 0.00 & 0.01 & 0.00 & 0.03 & $* * *$ & & \\
\hline 24 & 0.75 & 0.77 & 0.72 & 0.75 & 0.78 & 0.61 & 0.61 & 0.51 & 0.56 & 0.03 & 0.16 & 0.12 & 0.05 & 0.00 & 0.00 & 0.00 & 0.00 & 0.00 & 0.00 & 0.01 & 0.00 & 0.01 & 0.00 & $* * *$ & \\
\hline 25 & 0.75 & 0.76 & 0.73 & 0.75 & \begin{tabular}{|l|l|}
0.77 \\
\end{tabular} & 0.63 & 0.64 & 0.54 & 0.58 & 0.02 & 0.16 & 0.12 & 0.04 & 0.00 & 0.00 & 0.00 & 0.00 & 0.00 & 0.00 & 0.00 & 0.00 & 0.01 & 0.00 & 0.00 & $* * *$ \\
\hline
\end{tabular}

Table 3. Pairwise $\mathrm{N}_{\mathrm{ST}}$ values calculated between the analyzed populations of Siphonaria lessonii (20,000 iterations). Statistical significant differences are marked in bold. Where: 1) Iquique; 2) Antofagasta; 3) Punta de Choros; 4) Coquimbo; 5) Zapallar; 6) Pichilemu; 7) Puerto Saavedra; 8) La Misión; 9) Pucatrihue; 10) Putemún; 11) Melimoyu; 12) San Esteban Gulf; 13) Wager Island; 14) Port Famine; 15) Otway Sound; 16) Chabunco; 17) Porvenir; 18) Wulaia; 19) Lapataia; 20) San Julián; 21) Comodoro Rivadavia; 22) Puerto Madryn; 22) Mar del Plata; 23) La Pedrera,; 25) Hookers Point.

Quaternary glacial histories among the areas analyzed, as well as with the ecology of the species (i.e. habitat preferences, reproductive mode and bathymetric range). A concordance between historic biogeographic and contemporary phylogeographic patterns supports that evolutionary forces determining species distributions are also associated with spatial patterns of populations genetic structure ${ }^{5,6,18,44,45}$. Here we corroborate previous molecular results in the species ${ }^{24,28}$, and enhance our understanding of the Quaternary biogeography of the species by recognizing two clearly discriminated genetic clusters currently found in: 1) temperate areas across the Chile-Peru province, north of the $42^{\circ} \mathrm{S}$ in the Pacific, and 2) cold and temperate areas across the Magellan and Argentina provinces, respectively. The boundary of these clusters (C1 and C2) agrees with the $42^{\circ} \mathrm{S}$ biogeographic break demonstrated in several marine organisms ${ }^{36,46-50}$. This break represents the southern limit for many Chile-Peru Province species and the northern border for numerous Magellan ones ${ }^{13,36}$. The shift in species composition recorded in marine organisms at $42^{\circ} \mathrm{S}$ in the southeast Pacific has been explained by changes in salinity and wave exposure ${ }^{48,51}$, topographical shifts of the coastline as a consequence of the increased number of fjords and channels ${ }^{10,11,47}$, differences in main oceanographic patterns ${ }^{13,18,19,36,50}$, and Quaternary glaciological histories ${ }^{18,19,27,43}$. In fact, this biogeographic limit coincides with the separation of the West Wind Drift into two main Pacific currents, the Humboldt Current System flowing north across the Central Chilean coast and the Cape Horn Current heading south along the Pacific Magellan margin ${ }^{36,46}$. Our results support that $42^{\circ} \mathrm{S}$ represents a main boundary in the biogeography of $S$. lessonii in southern South America, as recorded in other marine invertebrates including patellogastropods ${ }^{14}$, polychaetes ${ }^{49}$ and macroalgae ${ }^{48}$.

Colonization toward former glacial areas involves a series of genetic bottlenecks, and accordingly recolonized areas should exhibit low genetic diversity dominated by few haplotypes and high frequency of sequences identical to or descended from the founding population ${ }^{1,4,6,38}$. The hypothesis predicting that non-glaciated areas should exhibit higher levels of genetic diversity and complexity than those sites that were heavily impacted during Quaternary glacial cycles is well supported here for the southeast Pacific, where populations from the Chile-Peru Province (R1) showed higher levels of genetic diversity than those located south of the $42^{\circ} \mathrm{S}$ break (R2), across areas that were heavily impacted by ice during glacial maxima. Thus the main phylogeographic signatures in $S$. lessonii, together with patterns of genetic diversity, structure, connectivity, and demographic reconstructions provide clear evidence in favor or the E-C model of Quaternary biogeography ${ }^{3}$. Nevertheless, contrary to our expectations, populations along the Atlantic margin of the species distribution, an area considered less impacted by ice 


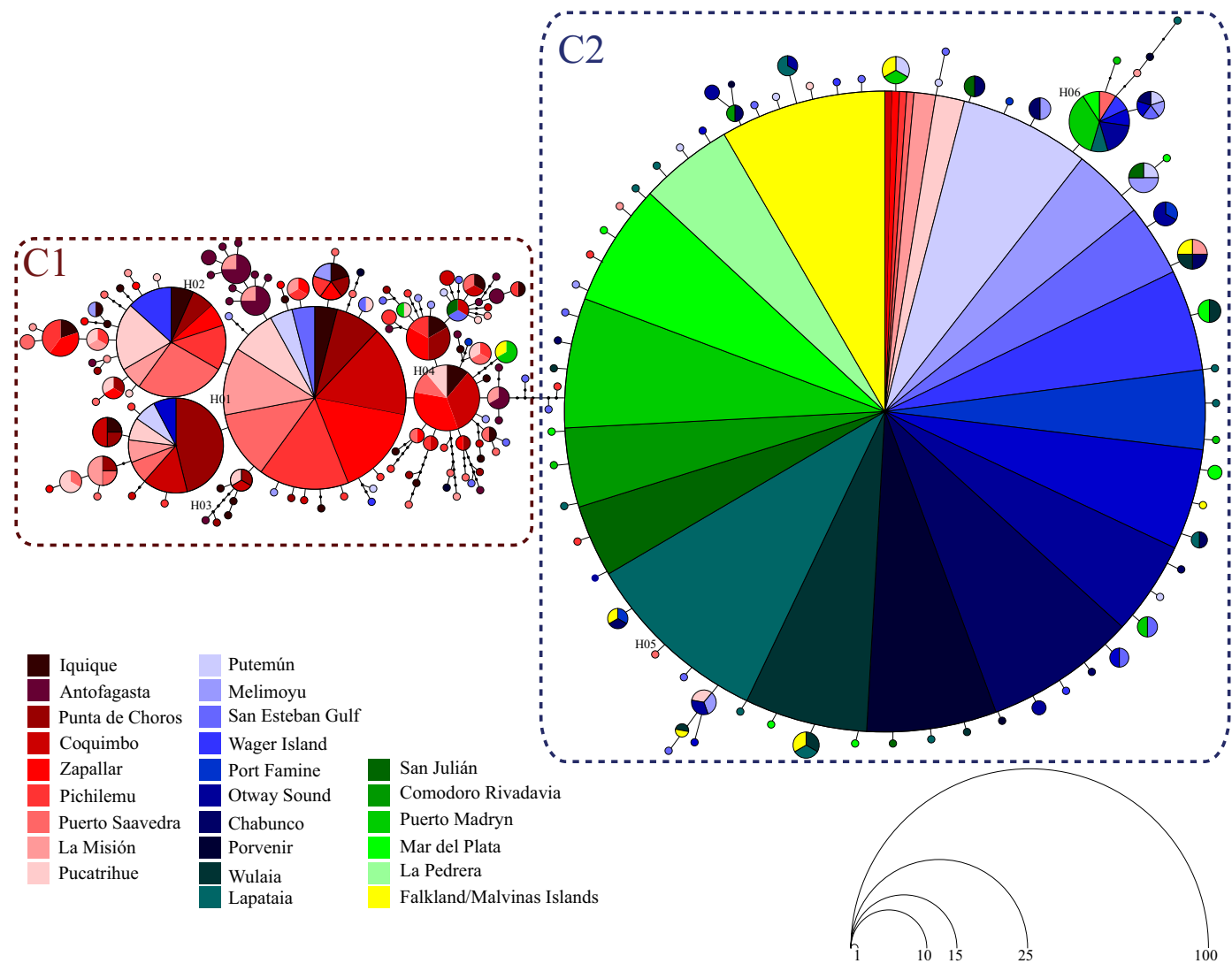

Figure 3. General maximum parsimony mtDNA haplotype network obtained from 625 Siphonaria lessonii sequences. Recorded haplotypes are represented by a colored circle indicating the locality where they were collected. The size of each circle is proportional to its frequency in the complete COI data set. For graphical purposes, main genetic clusters (C1 \& C2) are confined within dashed-line rectangles.

\begin{tabular}{|l|l|l|l|l|}
\hline Model & T.I. & LBF & Model prob & Model rank \\
\hline 1. full migration & -5274.456 & -367.701 & $<0.0001$ & 3 \\
\hline 2. C1 to C2 & $-\mathbf{5 0 9 0 . 6 0 5}$ & $\mathbf{0}$ & $\mathbf{1}$ & $\mathbf{1}$ \\
\hline 3. C2 to C1 & -5151.506 & -121.802 & $<0.0001$ & 2 \\
\hline 4. panmixia & -5659.569 & -1137.927 & $<0.0001$ & 4 \\
\hline
\end{tabular}

Table 4. Thermodynamic integration (T.I.) and log Bayes factor (LBF) comparisons for different migration models between the main genetic clusters recorded in Siphonaria lessonii (C1 and C2).

\begin{tabular}{|l|l|l|l|l|l|l|}
\hline Value & $\boldsymbol{\Theta}_{\mathbf{A}}$ & $\boldsymbol{\Theta} \mathbf{1}$ & $\mathbf{\Theta} \mathbf{2}$ & $\mathbf{t 0}$ (Years) & $\mathbf{m}_{\mathbf{1}>\mathbf{2}}$ & $\mathbf{m}_{2->1}$ \\
\hline HiPt & 357.5 & 128.5 & 21.5 & 211050.7246 & 0.135 & 0.245 \\
\hline Mean & 399.2 & 133.1 & 26.09 & 224094.2029 & 0.1682 & 0.2791 \\
\hline HPD95Lo & 218.5 & 89.5 & 8.5 & 156702.8986 & 0 & 0.075 \\
\hline HPD95Hi & 607.5 & 180.5 & 47.5 & 298007.2464 & 0.355 & 0.505 \\
\hline LRT & & & & & $2.583 \mathrm{~ns}$ & $\mathbf{1 0 . 0 9 2}$ **** \\
\hline
\end{tabular}

Table 5. Estimates of migration rates $(\mathrm{m})$ between the recorded Siphonaria lessonii's main genetic clusters in each direction, genetic diversities $(\Theta)$ of each group, ancestral diversity before splitting $\left(\Theta_{A}\right)$ and splitting time of both groups escalated in years. Analyses were based on the isolation-with-migration algorithm implemented in IMa2. High Point (HiPt), mean and 95\% highest posterior densities (95Lo and 95Hi) of the marginal posterior probabilities are shown. Significant patterns in asymmetrical migration rates were calculated using a Likelihood Ration Test (LTR). Significant values of $\mathrm{m}$ of the LRT are marked with asterisks: $* \mathrm{P}<0.05$, $* * \mathrm{P}<0.01, \mathrm{P}<0.001$. 

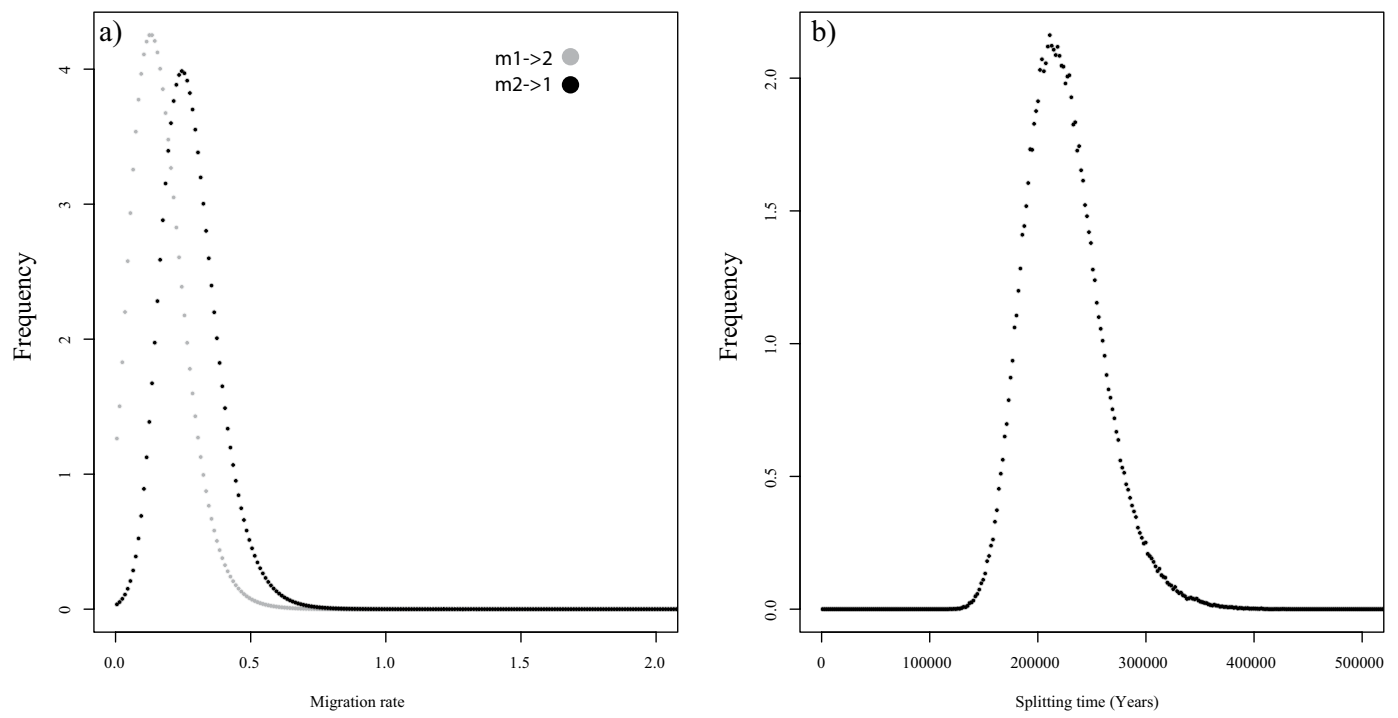

Figure 4. Isolation-with-migration analysis in Siphonaria lessonii. (a) Marginal posterior probability distribution of migration rates estimates between main genetic clusters C1 and C2. (b) Marginal posterior probability distribution of splitting time $(\mathrm{t})$ estimates between main genetic clusters $\mathrm{C} 1$ and $\mathrm{C} 2$ expressed in years. Splitting time was scaled into years using a $1 \%$ per million years substitution rate modified with a ten-fold correction following $\mathrm{Ho}^{39}$.
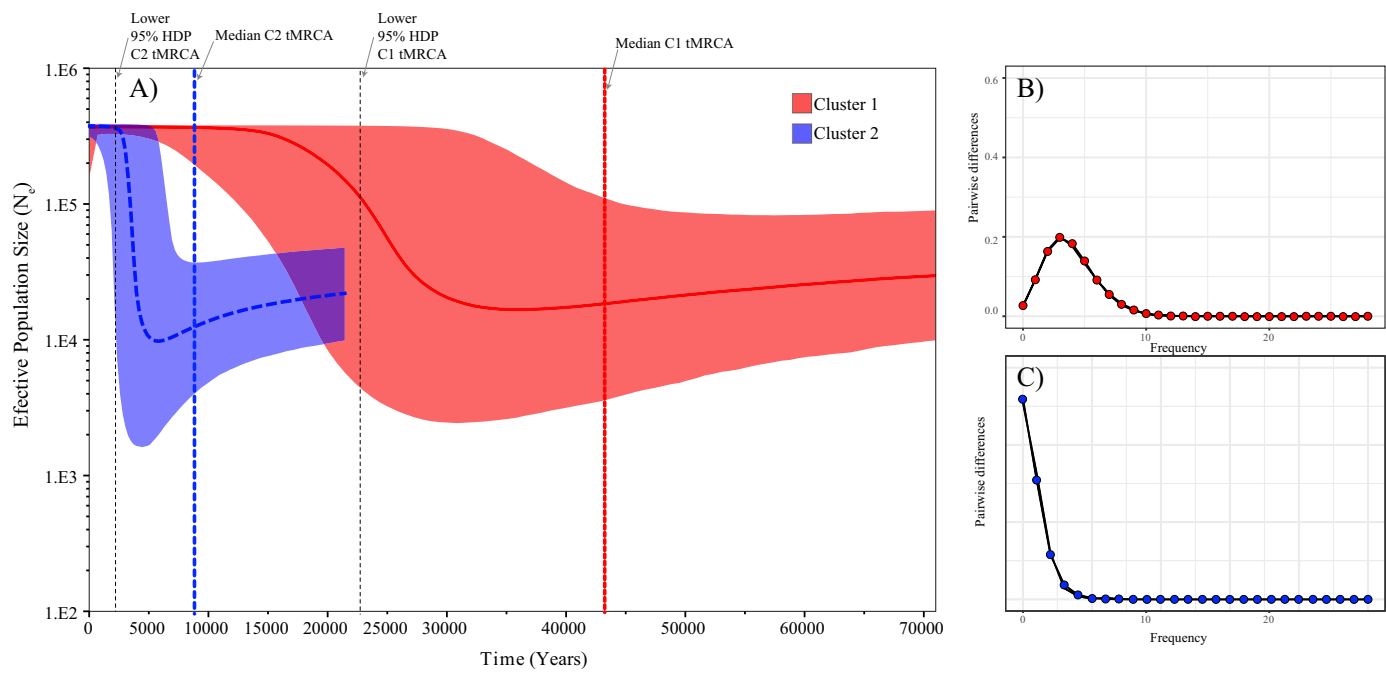

Figure 5. Demohraphic reconstruction in Siphonaria lessonii. (A) Bayesian Skyline plot mtDNA reconstructions of historical effective population sizes of Siphonaria lessonii across its distribution. The y-axis is the product of effective populations size $\left(\mathrm{N}_{\mathrm{e}}\right)$ and generation length in a log scale and the $\mathrm{x}$-axis in the time in years. The median estimate (red solid line for the Chile-Peru Province cluster and blue dashed line for the Magellan and Argentinean province cluster) and 95\% highest posterior density (HPD) limits (red for ChilePeru province and blue for Magellanic and Argentina provinces) are shown. The thick dashed lines represent the time of the most recent ancestor (tmrca) for the Chile-Peru province cluster (red) and for the MagellanicArgentina province cluster (blue). The thin dashed lines correspond to the lower 95\% HDP of the tmrca. Distributions of pairwise differences for the mtDNA gene COI in Siphonaria lessonii populations from (B) the Chile-Peru province (C1) and (C) the Magellan and Argentina provinces (C2). X-axis = Pairwise differences and $\mathrm{y}$-axis $=$ frequency.

during glacial periods, did not exhibit higher genetic diversity than those located within heavily glaciated areas in the Pacific margin of the Magellan province. Populations along temperate areas of the Argentinean province also showed low levels of genetic diversity and represent a continuity of the diversity found along the Magellan province. Absence of genetic differentiation between areas that were differentially affected by ice expansion and contraction along the Pacific and Atlantic margins of Magellan was also recorded in the pulmonate Siphonaria lateralis $^{21}$ and in the keystone patellogastropod Nacella magellanica ${ }^{20,23}$. 
During the LGM the sea level was between 120 and $140 \mathrm{~m}$ below its present level ${ }^{52}$; a large portion of the Atlantic Continental Shelf (ACS) was exposed as a consequence of these glacio-eustatic movements, with the development of enormous plains ${ }^{10}$. A plausible explanation for the genetic pattern recorded in S. lessonii and in other hard-bottom invertebrates across the Atlantic portion of their distributions relies on the fact that during the LGM the coastline lay 200 to $400 \mathrm{~km}$ away and was mainly dominated by depositional environments. Accordingly, it is likely that suitable hard substrate habitats for these species would have been strongly reduced and dominated by abrasion platforms of friable sedimentary rocks ${ }^{53}$. During the LGM, sandy environments dominated over the entire ACS while gravels and rocks were more abundant in southern areas ${ }^{54}$. Gravel environments covered around $12.5 \%$ of the total ACS and were very abundant south of $46^{\circ} \mathrm{S}$ off the coasts of Santa Cruz and Tierra del Fuego in Argentina and in the Malvinas/Falkland Islands. Bedrock outcrops during the LGM covered 2\% of the total ACS and also were more abundant in southern Atlantic areas of Magellan ${ }^{53}$. Accordingly, suitable refuges for S. lessonii would have been more available in the southern tip of the southwest Atlantic during the LGM, from where the species could have recolonized lower latitudes following the deglaciation process and the sea level increment, as proposed by Trovant et al. ${ }^{43,44}$ for the scorched mussel Perumytilus purpuratus. Thus current phylogeographic patterns in higher latitude near-shore marine invertebrates are not solely due to the impact of Quaternary glacial ice expansion and contraction but also to the availability of suitable habitats for the species.

Another interesting result recorded in S. lessonii is the absence of genetic structure along the Chile-Peru province (R1), as well as along the Magellan-Argentina provinces (R2). Such results may be a consequence of gene flow mediated by larval dispersal through the main flows of the Humboldt Current System, heading north along the Chilean coast, as well as the Cape Horn Current, heading south across the Magellan province, as recorded in several cold and temperate near-shore marine organisms ${ }^{19-21,55,56}$. Strong demographic and geographic expansion following post-glacial sea level rise may also be a suitable explanation for the low levels of genetic diversity and the high degree of genetic homogeneity in S. lessonii across these provinces. This pattern of genetic structure has been found in several Magellan marine benthic near-shore organisms with dispersive potential including invertebrates $^{20,55}$, vertebrates ${ }^{14,22}$ and macroalgae $e^{19,27}$. Finally, in contrast to recent phylogeographic analyses in sub-Antarctic invertebrates including Siphonaria species ${ }^{21}$, isopods ${ }^{57}$, and patellogastropods ${ }^{20,55}$, we found in $S$. lessonii a complete absence of genetic structure between continental Magellan and the Malvinas/Falkland Islands populations. The presence of a free-living dispersive stage in S. lessonii, absent in other sub-Antarctic Siphonaria species, seems to play a key role establishing genetic homogeneity across thousands of kilometers from Chiloé Island to Uruguay in the southwest Atlantic. Nevertheless, it is important to mention that the degree of resolution of the analyzed mitochondrial and nuclear markers does not allow us to distinguish whether the low divergence across these provinces are due to contemporary connectivity or is only a consequence of post-glacial demographic expansion.

Overall, our demographic time estimates (trmca and population expansions) should be tempered by the consideration of potential effects of three different sources of uncertainty including (a) evolutionary variability around the coalescence; (b) inadequate samplings, which may have missed some haplotypes and led to underestimates of population ages and (c) uncertainty of the molecular clock of the mtDNA COI ${ }^{16}$. Of these three, we recognize evolutionary variability around the coalescence as the main important source of uncertainty. Incomplete sampling of haplotypes is inevitable in any study of natural populations but the extensive geographical coverage we obtained for $S$. lessonii should ensure that most of the common haplotypes were included. Unsampled haplotypes are probably rare and terminal ones and should not likely affect the overall shape of the skyline plot or the isolation-with migration analyses. However, they may have some effect on the time of coalescence. Finally, the substitutional rate used in demographic inference analyses will also greatly determine the time estimation of population growth detected here. Nonetheless, even considering important confidence interval around the substitution rate used here, our estimations allow us to infer that divergence onset between clades and demographic events in S. lessonii were associated to Quaternary glacial processes and probably to the last glacial cycle. The paleogeographic evolution of the Magellan continental shelves (Pacific and Atlantic), together with genealogical and demographic reconstructions, as well as main patterns of genetic diversity/structure and gene flow analyses in S. lessonii allow us to propose a Quaternary biogeographic hypothesis for the species across its distribution in southern South America. At the end of the last interglacial period, the geographic distribution of S. lessonii was similar to that currently observed and extended from northern Chile to Uruguay. During glacial maxima, ice expansion over the Pacific Magellan coastline would have eradicated the species from this area, while populations in non-glaciated Pacific areas north of the $42^{\circ} \mathrm{S}$ break would have persisted along the Chile-Peru province. Along the Atlantic margin, a reduction of suitable habitats during glacial maxima would have led to local extinction of species associated with these hard bottom habitats, as recorded in other invertebrates during the Holocene such as the keystone Magellan gastropod Tegula atra ${ }^{58,59}$. As proposed by Aguirre et al. ${ }^{59}$ the absence of this important Magellan hard-bottom species along the Argentine Atlantic represents a climate-change driven shift associated with changes in Sea Surface Temperature (ca. $2^{\circ} \mathrm{C}$ higher), wind velocities (less), light (less), nutrient availability (less), and intensity of cold (less) and warm (increased) shallow water currents that altered water masses and biogeographical boundaries in the region. Paleo-eustatic reconstructions in the southwest Atlantic Patagonia suggest that during glacial maxima a higher proportion of suitable habitats for S. lessonii at higher latitudes in the Atlantic Magellanic margin ${ }^{53,54}$ may have allowed the persistence of small populations that acted as glacial refugia located close the southern tip of South America (Fig. 6A). At the end of the last glacial period, ice sheet contraction along Pacific Magellan shore and sea-level rise along the Atlantic margin would have uncovered new suitable habitats, allowing a stepwise recolonization towards lower latitudes (Fig. 6B) until S. lessonii recovered its present distribution (Fig. 6C). Separated since the onset of the last glacial period, populations isolated in the Atlantic refugia diverged from the genetic cluster recorded in the Chile-Peru province. Evidence of the separation of these clusters is the presence of at least six mutation steps between them, suggesting that they remained apart for a significant period of time. Following this, the southern clade (C2) re-expanded from the southern Atlantic 


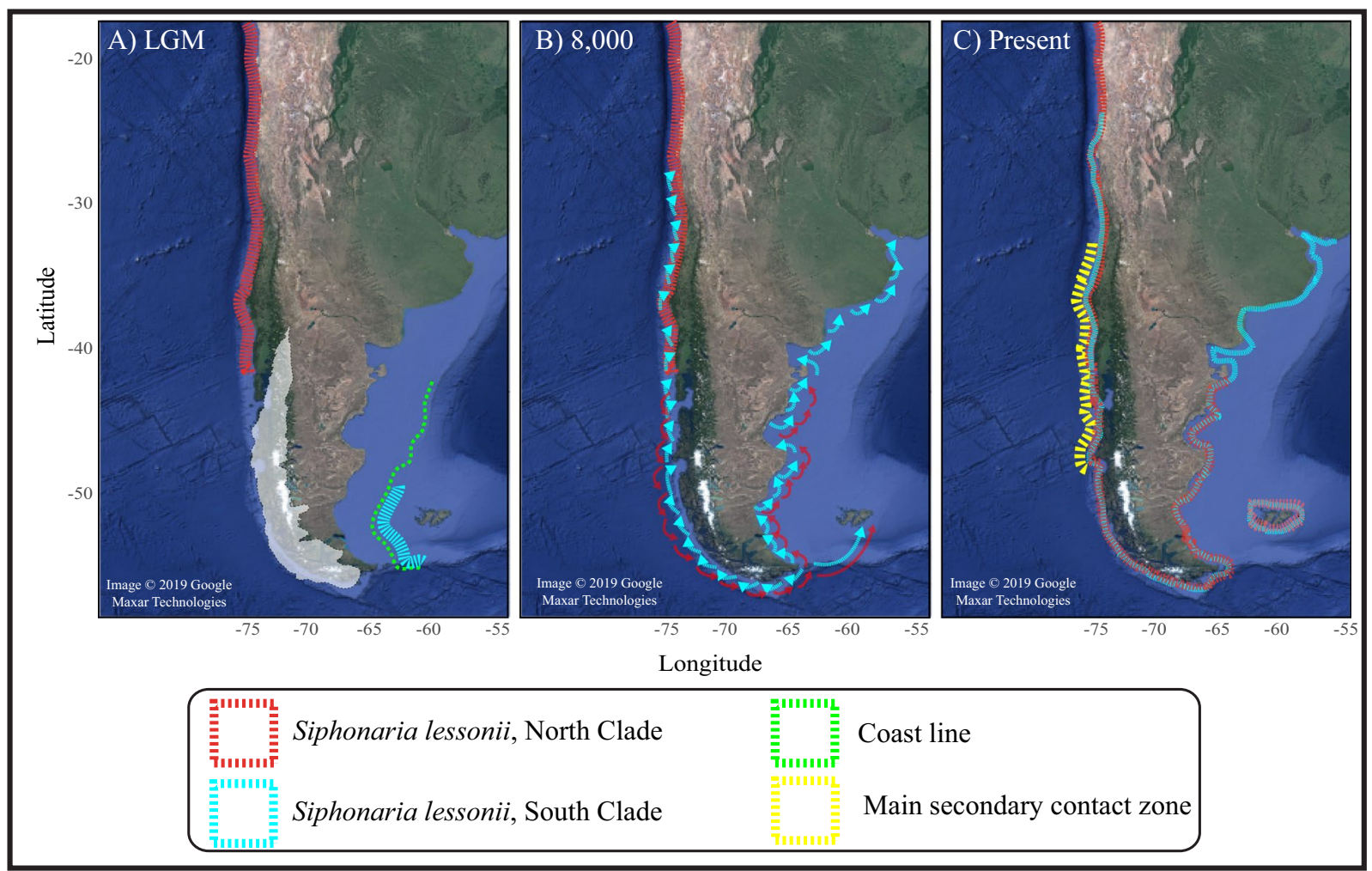

Figure 6. Hypothethical events leading to the current pattern of phylogeographic signal in Siphonaria lessonii across its distribution in southern South America. (A) During the Last Glacial Maximum (LGM) the northern clade was confined to warm-temperate regions of the southeastern Pacific while the southern clade was restricted to the cold-temperate Magellan province. (B) During the interglacial period the northern clade colonized the Magellan province while the southern clade expanded its distribution northward into the Argentina and westward to the Chile-Peru provinces, respectively. (C) Currently, both clades are broadly distribution in southern South America but a main secondary contact zone (SCZ) is recognized between the two clades. Map from Google earth Pro V 7.3.2.5776 (March 5, 2019). South America. - 37.9313여 $-69.7831^{\circ} \mathrm{W}$, Eye alt. $4210.9 \mathrm{Km}$. SIO, NOAA. Image Landset Copernicus. Data LDEO-Columbia, NSF, NOOA. http://www.earth.google.com [Dec 13, 2015].

margin towards the Pacific and to lower latitudes in the Atlantic following the deglaciation process around 8,000 years ago (Fig. 6B). Similarly, the northern clade (C1) expanded its distribution southward across the Magellan province to Puerto Madryn and the Malvinas/Falkland Islands in the Atlantic. Currently, the two main genetic clusters ( $\mathrm{C} 1$ and $\mathrm{C} 2$ ) recorded in $\mathrm{S}$. lessonii are broadly distributed across the species distribution but they are found in higher frequency between $34^{\circ} \mathrm{S}$ and $46^{\circ} \mathrm{S}$, producing a secondary contact zone with introgression when the clusters came back into contact (Figs. 6C and S2).

Phylogeographical studies in South American near-shore marine benthic organisms are helping us to understand the recent evolution of higher latitude marine biota. Main phylogeographic signatures in S. lessonii along the Pacific margin of its distribution provide obvious evidence towards the E-C model of Quaternary biogeography proposed by Provan \& Bennett ${ }^{3}$. In contrast, temperate area populations across the Argentina and Atlantic Patagonia showed very low levels of genetic diversity and structure and are clearly connected to the diversity recorded across the Pacific Magellan margin. Accordingly, the impact of Quaternary ice expansions over the demography of near-shore species does not solely explain current patterns of genetic diversity across the Atlantic Magellan margin and the Argentinean Province. Populations of S. lessonii across these areas that were less impacted by ice during glacial maxima share the diversity and structure that probably survived the last glacial maxima at higher latitudes refugia. Accordingly, ecological factors such as the availability of suitable habitats, bathymetry, developmental mode and the likelihood of dispersal through rafting are also key factors explaining main phylogeographical patterns in the species across its distribution in different South American ecoregions.

\section{Methods}

Sampling, DNA extraction, PCR amplification and sequencing. Individuals were sampled from 25 localities across the distribution of S. lessoni in the Chile-Peru Province (CP), the Magellan Province (MP), and the Argentina Province (AP) (Fig. 1; Table 1).

Specimens were preserved in ethanol (99\%) and DNA was prepared from the mantle using standard salting-out methodology following Aljanabi \& Martinez ${ }^{60}$. Universal primers LCO1490 and HCO2198 ${ }^{61}$ and its- $1 d$ and $i t s-4 r^{62}$ were used to amplify partial fragments of the mitochondrial gene cytochrome coxidase subunit I (COI) and the nuclear internal transcribed spacer region (ITS1 and ITS2), respectively. PCR procedures 
were performed using $2.5 \mu \mathrm{l}$ 10X Buffer (Invitrogen), $1.0 \mu \mathrm{l}$ of $50 \mathrm{mM} \mathrm{MgCl}$ (Invitrogen), $200 \mathrm{mM} \mathrm{dNTPs}$ (Invitrogen), $10 \mathrm{pg} / \mu \mathrm{l}$ each of dATP, dGTP, dCTP and dTTP (Invitrogen), $1 \mathrm{U}$ Taq polymerase (Invitrogen), $50 \mathrm{ng}$ of genomic DNA and $16.7 \mu \mathrm{l}$ of double-distilled water. Thermal cycling parameters for the analyzed markers followed González-Wevar et al. ${ }^{21}$. PCR products were purified using QIAquick Gel Extraction Kit (QIAGEN) and sequenced in both directions with an ABI3730 x 1 Automatic Sequencer at Macrogen Inc. (Seoul, South Korea).

Genetic diversity and population structure in S. lessonii. Mitochondrial (COI) and nuclear (ITS) data sets of S. lessonii were edited independently using GENEIOUS ${ }^{63}$ and aligned with MUSCLE ${ }^{64}$. New COI and ITS sequences have been deposited in GenBank under the following accession numbers: MN436071-MN436695 (COI), and MN436696-MN436714 (ITS). In order to evaluate how saturation of transitions is accumulated in relation to divergence we performed a saturation analysis in DAMBE $5^{65}$. Following Wright ${ }^{66}$, we evaluated the codon usage of mtDNA sequences using the effective number of codon value (ENC) in DNASP v. $5^{67}$.

Polymorphism of mtDNA sequences was estimated in DNASP using standard diversity indices: number of haplotypes $(k)$, segregating sites $(S)$, haplotype diversity $(H)$, average number of pairwise differences $(\Pi)$, and nucleotide diversity $(\pi)$ per site, genetic cluster, and for the whole COI data set.

Levels of genetic structure between populations of $S$. lessonii were estimated following Pons and Petit ${ }^{68}$ using main pairwise differences $\left(\mathrm{N}_{\mathrm{ST}}\right)$ and haplotype frequencies $\left(\mathrm{G}_{\mathrm{ST}}\right)$ in ARLEQUIN v. 3.5 ${ }^{69}$. The statistical significance of pairwise population comparisons was determined through permutation tests (20,000 iterations) of haplotype identities following Excoffier et al. ${ }^{69}$. We also determined levels of phylogeographic differentiation using the nearest-neighbor statistic $\left(\mathrm{S}_{\mathrm{nn}}\right)$ in DNASP and the statistical significance of the analyses was determined using a permutation test (10,000 iterations) following Hudson ${ }^{70}$.

The spatial mtDNA genetic structure of $S$. lessonii across its distribution was inferred using two clustering methods. First, through a Bayesian model we determined the number and composition of panmictic groups and the spatial boundaries among them using GENELAND v. 2.0.0 $0^{71}$ in the R environment (R, version 2.4.1) $)^{72}$. The best association of localities was estimated using a Markov Chain Monte Carlo (MCMC) procedure including genetic and geographic information following Guillot et al. ${ }^{71}$. Analyses were run using $50 \times 10^{6} \mathrm{MCMC}$ iterations sampled each 1,000 steps. Stationarity of the analyses was identified in TRACER v.1.5 (http://beast.bio. ed.ac.uk/Tracer) and $10 \%$ of the iterations were removed as burn-in. A maximum number of clusters $(\mathrm{K}=24)$ was included in the analyses to determine model parameters and posterior probabilities of group membership. Second, using SAMOVA we determined the composition and number of geographically homogeneous and maximally differentiated groups of localities following Dupanloup et al. ${ }^{73}$. This analysis allows characterization of spatial genetic structure by separating the variance into within populations, among populations within groups and among groups. This methodology seeks to maximize the proportion of total genetic variance due to differences among groups.

Genealogical reconstructions, gene flow and connectivity in S. lessonii. Genealogical relationships in $S$. lessonii were constructed based on mtDNA (COI) and nucDNA (ITS) sequences using maximum parsimony networks in HAPVIEW ${ }^{74}$. Different models of gene flow between the mtDNA groups were tested for different scenarios using the software MIGRATE v.3.5 following Beerli \& Felsenstein ${ }^{75}$. Four candidate models were compared constraining the presence of directionality of gene flow between the genetic groups found in S. lessonii. A first model permitted bidirectional gene flow (full-island model). Models 2 and 3 were defined based on the asymmetry of gene flow between groups. Finally, the fourth model assumed the groups were a single panmictic population. The analyses were performed using a $\mathrm{TrN}+\mathrm{I}+\mathrm{G}$ substitution model and transition-transversion ratio of 16.9927, estimated using JMODELTEST v $2^{76}$. The specific substitution rate for the selected marker in siphonariids ${ }^{77,78}$, was set to constant, following Darriba et al. ${ }^{76}$. The analysis included a long chain with 500,000 recorded parameter steps with a sampling interval of 100 and a burn-in of $10 \%$. Following Darriba et al. ${ }^{76}$ we used a heated scheme of $1.0,1.5,3.0$. and 1000000.0 to estimate the marginal likelihoods of the models to compared them. A thermodynamic integration approximation (T.I.) was used for the log-equivalent Bayes Factor (LBF) analyses following Beerli \& Palczewski ${ }^{79}$. The associated probability of each model in relation to another was estimated through Bayes Factors following Kass and Rafery ${ }^{80}$. We also determined different demographic indices to evaluate asymmetric isolation-with-migration models between the recognized genetic groups using IMA2 software ${ }^{39,81}$. For this we performed several preliminary runs in the M-mode (Markov Chain Monte Carlo; MCMC mode) to estimate the best set of priors. Uniform priors were used to determine the effective population size $\left(\Theta_{1}, \Theta_{2}\right.$, and ancestral $\left.\Theta_{a}, \Theta=600\right)$ and splitting time $(t=30)$; an exponential prior $(m e a n=20)$ for gene flow (m) was assumed. We performed $80 \times 10^{6} \mathrm{MCMC}$ steps sampling every 100 generations, with a burn-in period of $10 \%$. Mitochondrial sequences in S. lessonii were assumed to mutate under the HKY mutation model following Hey and Nielsen ${ }^{81}$. Simulated genealogies were performed under the L-Mode (Load Tree mode) to determine the log maximum likelihood and credibility interval (95\% under HPD) estimates for migration parameters using a likelihood ratio test. Finally, under the L-Mode mtDNA data set of $S$. lessonii was compared against a null model of no migration ${ }^{39,81}$.

Demographic inference in S. lessonii. Demographic mtDNA dynamics were determined using a Bayesian skyline plot (BSP) methodology implemented in the program BEAST v.1.8 ${ }^{82}$. For this we performed three independent Bayesian Monte Carlo analyses for $100 \times 10^{6}$ generations. Trees and parameters were sampled every 1,000 generations in each of the genetic groups of $S$. lessonii. Previously, JMODELTEST was used to estimate the proper substitution model using the Akaike information criterion (AIC). Following Ho et al. ${ }^{83}$ we used a rough tenfold evolutionary rate correction for population-based analyses. A time-dependency rate of molecular evolution has been described where the short-term mutation rate ( 1 to $2 \mathrm{Ma}$ ) could be tenfold higher than the long-term substitution rate used for phylogenetic reconstructions. Accordingly, BSP analyses 
used a $10 \%$ population-based mutation rate considering the $1.0 \%$ phylogenetic rate used for siphonariids ${ }^{77,78}$. Convergence between runs was confirmed with TRACER and the results for multiple runs were combined using LOGCOMBINER v1.4. $7^{84}$.

We performed statistical neutrality tests (Tajima's D and Fu's $\mathrm{F}_{\mathrm{S}}$ ) using DNASP for the whole COI data set and for each recognized genetic group to estimate whether sequences deviate from mutation-drift equilibrium. Demographic reconstructions were also estimated using the comparison of pairwise differences between haplotypes (mismatch distribution) for each recognized group to the expected distribution under the sudden expansion growth model ${ }^{85}$. For this we estimated three main parameters: i) $\tau=$ date of population expansion/ contraction measured in units of mutational time $(\tau=2 \mu \mathrm{t})$ where $\mathrm{t}=$ time in years and $\mu=$ mutational rate per sequence per year, ii) initial theta $\theta_{i}=2 N_{i} \mu$ before the population expansion/contraction), and iii) final theta $\theta_{t}=2 N_{t} \mu$ after population expansion/contraction. Finally, demographic parameters were calculated in DNASP using a nonlinear least square approach following Schneider \& Excoffier ${ }^{86}$.

\section{Data availability}

New COI and ITS sequences in Siphonaria lessonii will be available in GenBank under Accession Numbers MN436071-MN436695 (COI), and MN436696-MN436714 (ITS1 \& ITS2).

Received: 15 April 2019; Accepted: 28 December 2019;

Published online: 21 January 2020

\section{References}

1. Hewitt, G. The genetic legacy of the Quaternary ice ages. Nature 405, 907-913 (2000).

2. Stewart, J. R., Lister, A. M., Barnes, I. \& Dalén, L. Refugia revisited: individualistic responses of species in space and time. P. Roy. Soc. B-Biol. Sci. 277, 661-671 (2010).

3. Provan, J. \& Bennett, K. D. Phylogeographic insights into cryptic glacial refugia. Trends Ecol. Evol. 23, 564-571 (2008).

4. Hewitt, G. M. Post-glacial re-colonization of European biota. Biol. J. Linn. Soc. 68, 87-112 (1999).

5. Maggs, C. A. et al. Evaluating signatures of glacial refugia for North Atlantic benthic marine taxa. Ecology 89, S108-S122 (2008).

6. Marko, P. B. et al. The "Expansion-Contraction" model of Pleistocene biogeography: rocky shores suffer a sea change? Mol. Ecol. 19, 146-169 (2010)

7. González-Wevar, C. A. et al. Extinction and recolonization of maritime Antarctica in the limpet Nacella concinna (Strebel, 1908) during the last glacial cycle: toward a model of Quaternary biogeography in shallow Antarctic invertebrates. Mol. Ecol. 22, 5221-5236 (2013).

8. Hewitt, G. M. Genetic consequences of climatic oscillations in the Quaternary. Philos. T. R. Soc. B. 359, 183-195 (2004).

9. Birks, H. J. B. Some reflections on the refugium concept and its terminology in historical biogeography, contemporary ecology and global-change biology. Biodiversity 16, 196-212 (2015).

10. Rabassa, J., Coronato, A. \& Martínez, O. Late Cenozoic glaciations in Patagonia and Tierra del Fuego: an updated review. Biol. J. Linn. Soc. 103, 316-335 (2011).

11. Hulton, N. R. J., Purves, R. S., McCulloch, R. D., Sugden, D. E. \& Bentley, M. J. The last glacial maximum and deglaciation in southern South America. Quat. Sci. Rev. 21, 233-241 (2002).

12. Hein, A. S. et al. The chronology of the Last Glacial Maximum and deglacial events in central Argentine Patagonia. Quat. Sci. Rev. 29, 1212-1227 (2010).

13. Valdovinos, C., Navasrrete, S. A. \& Marquet, P. A. Mollusk species diversity in the Southern Pacific: why are there more species towards the pole? Ecography 26, 139-144 (2003).

14. González-Wevar, C. A. et al. Following the Antarctic Circumpolar Current: patterns and processes in the biogeography of the limpet Nacella (Mollusca: Patellogastropoda) across the Southern Ocean. J. Biogeogr. 44, 861-874 (2017).

15. Glasser, N. \& Jansson, K. The Glacial Map of southern South America. J. Maps 4, 175-196 (2008).

16. Ruzzante, D. E. et al. Phylogeography of the Percichthyidae (Pisces) in Patagonia: roles of orogeny, glaciation, and volcanism. Mol. Ecol. 15, 2949-2968 (2006).

17. Zemlak, T. S. et al. Across the southern Andes on fin: glacial refugia, drainage reversals and a secondary contact zone revealed by the phylogeographical signal of Galaxias platei in Patagonia. Mol. Ecol. 17, 5049-5061 (2008).

18. González-Wevar, C. et al. Contrasting genetic structure and diversity of Galaxias maculatus (Jenyns, 1848) along the Chilean coast: stock identification for fishery management. J. Hered. 106, 439-447 (2015).

19. Fraser, C. I., Thiel, M., Spencer, H. G. \& Waters, J. M. Contemporary habitat discontinuity and historic glacial ice drive genetic divergence in Chilean kelp. BMC Evol. Biol. 10, 203b (2010).

20. González-Wevar, C. A. et al. Towards a model of postglacial biogeography in shallow marine species along the Patagonian Province: lessons from the limpet Nacella magellanica (Gmelin, 1791). BMC Evol. Biol. 12, 139 (2012).

21. González-Wevar, C. A. et al. Unexpected absence of island endemics: Long-distance dispersal in higher latitude sub-Antarctic Siphonaria (Gastropoda: Euthyneura) species. J. Biogeogr. 45, 874-884 (2018).

22. Ceballos, S. G., Lessa, E. P., Licandeo, R. \& Fernández, D. A. Genetic relationships between Atlantic and Pacific populations of the notothenioid fish Eleginops maclovinus: the footprints of Quaternary glaciations in Patagonia. Heredity 116, 372-377 (2015).

23. de Aranzamendi, M. C., Bastida, R. \& Gardenal, C. N. Genetic population structure in Nacella magellanica: Evidence of rapid range expansion throughout the entire species distribution on the Atlantic coast. J. Exper. Mar. Biol. Ecol. 460, 53-61 (2014).

24. Nuñez, J. D., Fernández Iriarte, P. J., Ocampo, E. H., Iudica, M. \& Cledón, M. Deep phylogeographic divergence among populations of limpet Siphonaria lessoni on the east and west coasts of South America. Mar. Biol. 162, 595-605 (2015).

25. Ruzzante, D. E. \& Rabassa, J. Palaeogeography and palaeoclimatology of Patagonia: effects on biodiversity. Biol. J. Linn. Soc. 103, 221-228 (2011)

26. Jakob, S. S., Martinez-Meyer, E. \& Blattner, F. R. Phylogeographic analyses and paleodistribution modeling indicate Pleistocene In Situ survival of Hordeum species (Poaceae) in Southern Patagonia without genetic or spatial restriction. Mol. Biol. Evol. 26, 907-923 (2009).

27. Macaya, E. C. \& Zuccarello, G. C. Genetic structure of the giant kelp Macrocystis pyrifera along the southeastern Pacific. Mar. Ecol-Prog. Ser. 420, 103-112 (2010).

28. Pardo-Gandarillas, M. C. et al. Phylogeography and species distribution modelling reveal the effects of the Pleistocene ice ages on an intertidal limpet from the south-eastern Pacific. J. Biogeogr. 45, 1751-1767 (2018).

29. Trovant, B. et al. Scorched mussels (BIVALVIA: MYTILIDAE: BRACHIDONTINAE) from the temperate coasts of South America: Phylogenetic relationships, trans-Pacific connections and the footprints of Quaternary glaciations. Mol. Phylogenet. Evol. 82, 60-74 (2015). 
30. Azpilicueta, M. M., Marchelli, P. \& Gallo, L. A. The effects of Quaternary glaciations in Patagonia as evidenced by chloroplast DNA phylogeography of Southern beech Nothofagus obliqua. Tree Genet. Genomes 5, 561-571 (2009).

31. Trovant, B., Ruzzante, D. E., Basso, N. G. \& Orenzans, J. M. (Lobo). Distinctness, phylogenetic relations and biogeography of intertidal mussels (Brachiodontes, Mytilidae) from the south-western. Atlantic. J. Mar. Biol. Ass. UK 93, 1843-1855 (2013).

32. Dayrat, B., Goulding, T. C. \& White, T. R. Diversity of Indo-West Pacific Siphonaria (Mollusca: Gastropoda: Euthyneura). Zootaxa 3779, 246-276 (2014).

33. Güller, M., Zelaya, D. G. \& Ituarte, C. How many Siphonaria species (Gastropoda: Euthyneura) live in southern South America? J. Mollus. Stud. 82, 80-96 (2015).

34. de Castellanos, Z. A. J., Landoni, N. \& Dadon, J. Catálogo descriptivo de la malacofauna marina magallánica - Opistobranchia (excepto Nudibranchia y Pulmonata). La Plata: Comisión de Investigaciones Científicas Provincia de Buenos Aires. 37pp (1993).

35. Spalding, M. D. et al. Marine ecoregiones of the World: a bioregionalization of coastal and shelf areas. Bioscience 57, 573-583 (2007).

36. Camus, P. Biogeografía marina de Chile continental. Rev. Chil. Hist. Nat. 74, 587-617 (2001).

37. Zabala, S., Averbuj, A., Penchaszadeh, P. E. \& Bigatti, G. Early development of the limpet Siphonaria lessonii Blainville, 1827 in populations affected by different physical stressors. Mar. Biol. Res. 14, 148-157 (2018).

38. Posada, D. \& Crandall, K. A. Intraspecific gene genealogies: trees grafting into networks. Trends Ecol. Evol. 16, 37-45 (2001).

39. Nielsen, R. \& Wakeley, J. Distinguishing migration from isolation: a Markov chain Monte Carlo approach. Genetics 158, 885-896 (2001).

40. Parmesan, C. Ecological and evolutionary responses to recent climate change. Annu. Rev. Ecol. Evol. S. 37, 1-35 (2006).

41. Davis, M. B. \& Shaw, R. G. Range shifts and adaptive responses to Quaternary climate change. Science 292, 673-679 (2004),

42. Allcock, A. L. \& Strugnell, J. M. Southern Ocean diversity: new paradigms from molecular ecology. Trends Ecol. Evol. 27, 520-528 (2012).

43. Fraser, C. I., Nikula, R., Ruzzante, D. E. \& Waters, J. M. Poleward bound: biological impacts of Southern Hemisphere glaciation. Trends Ecol. Evol. 27, 462-471 (2012).

44. Waters, J. M. \& Roy, M. S. Out of Africa: The slow train to Australasia. Syst. Biol. 53, 18-24 (2004).

45. Haye, P. A. et al. Phylogeographic structure in benthic marine invertebrates of the Southeaster Pacific coast of Chile with differing dispersal potential. PLoS ONE, 9, e88613 (2014).

46. Brattström, H. \& Johanssen, A. Ecological and regional zoogeography of the marine benthic fauna of Chile. Sarsia 68, 289-339 (1983).

47. Lancellotti, D. A. \& Vasquez, J. A. Zoogeografía de macroinvertebrados bentónicos de la costa de Chile: contribución para la conservación marina. Rev. Chil. Hist. Nat. 73, 99-129 (2000).

48. Meneses, I. \& Santelices, B. Patterns and breaking points in the distribution of benthic algae along the temperate Pacific coast of South America. Rev. Chil. Hist. Nat. 73, 615-623 (2000).

49. Hernández, C. E., Moreno, R. A. \& Rozbaczylo, N. Biogeographical patterns and Rapoport's rule in southeastern Pacific benthic polychaetes of the Chilean coast. Ecography 28, 363-373 (2005)

50. Ewers-Saucedo, C. et al. The oceanic concordance of phylogeography and biogeography: a case study in Notochthalamus. Ecol. Evol. 6, 4403-4420 (2016).

51. Escribano, R., Fernández, M. \& Aranís, A. Physical-chemical processes and patterns of diversity of the chilean eastern boundary pelagic and benthic marine ecosystems: an overview. Gayana 67, 190-205 (2003).

52. Fleming, K. et al. Refining the eustatic sea level curve since the Last Glacial Maximum using far- and intermediate-field sites. Earth Planet. Sc. Lett. 163, 327-342 (1998).

53. Ponce, J. F., Rabassa, J., Coronato, A. \& Borromei, A. M. Palaeogeographical evolution of the Atlantic coast of Pampa and Patagonia from the last glacial maximum to the Middle Holocene. Biol. J. Linn. Soc. 103, 363-379 (2011).

54. Violante et al. The Argentine continental shelf: morphology, sediments, processes and evolution since the Last Glacial Maximum in Continental Shelves of the World: their evolution during the Last Glacio-Eustatic Cycle (ed. Chiocci, F. L. \& Chivas, A. R.) 55-68 (The Geological Society of London, 2014).

55. González-Wevar, C. A. et al. Genetics, gene flow, and glaciation: The case of the South American limpet Nacella mytilina. PLoS ONE 11, e0161963 (2016).

56. Hüne, M. et al. Low levels of genetic divergence between Harpagifer fish species (Perciformes: Notothenioidei) suggests a Quaternary colonization of Patagonia from the Antarctic Peninsula. Polar Biol. 38, 607-617 (2015).

57. Leese, F., Kop, A., Wägele, J. -W. \& Held, C. Cryptic speciation in a benthic isopod from Patagonian and Falkland Island waters and the impact of glaciations on its population structure. Front. Zool. 5, 1-15 (2008).

58. Aguirre, M. L., Richiano, S., Donato, M. \& Farinati, E. A. Tegula atra (Lesson, 1830) (Mollusca, Gastropoda) in the marine Quaternary of Patagonia (Argentina, SW Atlantic): Biostratigraphical tool and palaeoclimate-palaeoceanographical signal. Quatern. Int. 305, 163-187 (2013).

59. Charó, M. P., Gordillo, S., Fucks, E. E. \& Giaconi, L. M. Late Quaternary molluscs from the northern San Matías Gulf (Northern Patagonia, Argentina), southwestern Atlantic: Faunistic changes and paleoenvironmental interpretation. Quatern. Int. 352, 26-47 (2014).

60. Aljanabi, S. M. \& Martinez, I. Universal and rapid salt-extraction of high quality genomic DNA for PCR-based techniques. Nucleic Acids Res. 25, 4692-4693 (1997).

61. Folmer, O., Black, M., Hoeh, W., Lutz, R. \& Vrijenhoek, R. DNA primers for amplification of mitochondrial cytochrome c oxidase subunit I from diverse metazoan invertebrates. Mol. Mar. Biol. Biotechnol. 3, 294-299 (1994).

62. Oliverio, M. \& Mariottini, P. Contrasting morphological and molecular variation in Coralliophila meyendorffii (Muricidae, Coralliophilinae). J. Mollusc. Stud. 67, 243-246 (2001).

63. Kearse, M. et al. Geneious Basic: An integrated and extendable desktop software platform for the organization and analysis of sequence data. Bioinformatics 28, 1647-1649 (2012).

64. Edgar, R. C. MUSCLE: a multiple sequence alignment method with reduced time and space complexity. BMC Bioinformatics 5, 113 (2004).

65. Xia, X. DAMBE5: A comprehensive software package for data analysis in Molecular Biology and Evolution. Mol. Biol. Evol. 30, $1720-1728$ (2013).

66. Wright, $\mathrm{F}$. The effective number of codons' used in a gene. Gene 87, 23-29 (1990).

67. Librado, P. \& Rozas, J. DnaSP v5: a software for comprehensive analysis of DNA polymorphism data. Bioinformatics 25, 1451-1452 (2009).

68. Pons, O. \& Petit, R. J. Measuring and testing genetic differentiation with ordered Versus unordered alleles. Genetics 144, 1237-1245 (1996).

69. Excoffier, L., Laval, G. \& Schneider, S. Arlequin (version 3.0): An integrated software package for population genetics data analysis. Evol. Bioinform. Online 1, 37-50 (2005).

70. Hudson, R. R. A new statistic for detecting genetic differentiation. Genetics 155, 2011-2014 (2000).

71. Guillot, G., Mortimer, F. \& Estoup, A. Geneland: a computer package for landscape genetics. Mol. Ecol. Notes 5, 712-715 (2005).

72. Ihaka, R. \& Gentleman, R. R: A language for data analysis and graphics. J. Comput. Graph. Stat. 5, 299-314 (1996).

73. Dupanloup, I., Schneider, S. \& Excoffier, L. A simulated annealing approach to define the genetic structure of population. Mol. Ecol. 11, 2571-2581 (2002). 
74. Salzburger, W., Ewing, G. B. \& von Haeseler, A. The performance of phylogenetic algorithms in estimating haplotype genealogies with migration. Mol. Ecol. 20, 1952-1963 (2011).

75. Beerli, P. \& Felsenstein, J. Maximum likelihood estimation of a migration matrix and effective population sizes in $\mathrm{n}$ subpopulations by using a coalescent approach. P. Natl. Acad. Sci. USA 98, 4563-4568 (2001).

76. Darriba, D., Taboada, G. L., Doallo, R. \& Posada, D. jModelTest 2: more models, new heuristics and parallel computing. Nat. Methods 9,772-772 (2012).

77. Teske, P. R. et al. Climate-driven genetic divergence of limpets with different life histories across a southeast African marine biogeographic disjunction: different processes, same outcome. Mol. Ecol. 20, 5025-5041 (2011).

78. Wang, J., Tsang, L. M. \& Dong, Y. -W. Causations of phylogeographic barrier of some rocky shore species along the Chinese coastline. BMC Evol. Biol. 15, 114 (2015).

79. Beerli, P. \& Palczewski, M. Unified framework to evaluate panmixia and migration direction among multiple sampling locations. Genetics 185, 313-326 (2010).

80. Kaas, R. E. \& Raftery, A. E. Bayes Factors. J. Am. Stat. Assoc. 90, 773-795 (1995).

81. Hey, J. \& Nielsen, R. Integration within the Felsenstein equation for improved Markov chain Monte Carlo methods in population genetics. P. Natl. Acad. Sci. USA 104, 2785-2790 (2007).

82. Drummond, A. J., Suchard, M. A., Xie, D. \& Rambaut, A. Bayesian Phylogenetics with BEAUti and the BEAST 1.7. Mol. Biol. Evol. 29, 1969-1973 (2012).

83. Ho, S. Y. W. et al. Time-dependent rates of molecular evolution. Mol. Ecol. 20, 3087-3101 (2011).

84. Drummond, A. J. \& Rambaut, A. BEAST: Bayesian Evolutionary Analysis by Sampling Trees. BMC Evol. Biol. 7, 214 (2007).

85. Rogers, A. R. \& Harpending, H. Population growth makes waves. Mol. Biol. Evol. 9, 552-569 (1992).

86. Schneider, S. \& Excoffier, L. Estimation of past demographic parameters from a distribution of pairwise differences when the mutation rates vary among sites: Application to human mitochondrial DNA. Genetics 152, 1879-1889 (1999).

\section{Acknowledgements}

Several projects and institutions are greatly appreciated, including a Fondecyt Initiation Project 11140087, the program FONDAP IDEAL, Project 15150003 and the INACH project RG_18-17 to C. A. G-W. We also appreciate the support of the projects: P05-002 ICM, PFB 023 (IEB), project GAB (ACT172065) and Fondecyt Regular Project 1151336 to E. P and C. A. G-W.; project UMAG 027210, PR-06CRN-18 to C. A. G-W. and S. R; projects PIP 798 (2012-2014) and PIP 770 (2015-2017) (CONICET) and 15/E725 (UNMdP) to P. F. I. and J. D. N. There were no additional external funds related to this study. Specimens were sampled under the Chilean legislation (Technical Memorandum P. INV N ${ }^{\circ} 429 / 2015$ SUBPESCA). Permits to collect specimens in Falkland Islands were issued by the Environmental Planning Department, the Falkland Islands Government. Authors would also like to thank J. Naretto, P. Brickle, J. Ojeda, K. Gérard, L. Porrini and A. Garese for assistance during field works. Samples from Uruguay were delivered by Dr. F. Scarabino from the Centro Universitario Regional del Este-Cure, Sede Rocha, Universidad de la República, Uruguay. We recognize the SCAR, AntEco and EBA programs, CAML and SAERI, for encouraging and supporting Antarctic and sub-Antarctic biogeographic studies. This manuscript contributes to the SCAR AntEco programme.

\section{Author contributions}

C.A.G.-W., E.P., P.J.F.I., N.I.S. and P.A.H. conceived the idea and designed the analyses. C.A.G.-W., J.D.N., S.R., M.H., L.F. and N.I.S. collected specimens from different localities across the species' distribution. C.A.G.-W., J.D.N. and N.I.S., performed molecular experiments. C.A.G.-W. and N.I.S. performed the analyses of the recorded data. P.J.F.I., C.A.G.-W., N.I.S., P.A.H. and E.P., wrote the paper. C.A.G.-W., N.I.S., P.J.F.I. and E.P. contributed intellectually to the interpretation and discussion of results. All authors read and approved the final version of the manuscript.

\section{Competing interests}

The authors declare no competing interests.

\section{Additional information}

Supplementary information is available for this paper at https://doi.org/10.1038/s41598-020-57543-4.

Correspondence and requests for materials should be addressed to C.A.G.-W.

Reprints and permissions information is available at www.nature.com/reprints.

Publisher's note Springer Nature remains neutral with regard to jurisdictional claims in published maps and institutional affiliations.

(c) (1) Open Access This article is licensed under a Creative Commons Attribution 4.0 International (c) License, which permits use, sharing, adaptation, distribution and reproduction in any medium or format, as long as you give appropriate credit to the original author(s) and the source, provide a link to the Creative Commons license, and indicate if changes were made. The images or other third party material in this article are included in the article's Creative Commons license, unless indicated otherwise in a credit line to the material. If material is not included in the article's Creative Commons license and your intended use is not permitted by statutory regulation or exceeds the permitted use, you will need to obtain permission directly from the copyright holder. To view a copy of this license, visit http://creativecommons.org/licenses/by/4.0/.

(C) The Author(s) 2020 